\title{
Ordinal Regression Models in Psychology: A Tutorial
}

\author{
Paul-Christian Bürkner ${ }^{1} \&$ Matti Vuorre ${ }^{2}$ \\ ${ }^{1}$ Department of Psychology, University of Münster, Germany \\ 2 Department of Psychology, Columbia University, USA
}

\begin{abstract}
Ordinal variables, while extremely common in Psychology, are almost exclusively analysed with statistical models that falsely assume them to be metric. This practice can lead to distorted effect size estimates, inflated error rates, and other problems. We argue for the application of ordinal models that make appropriate assumptions about the variables under study. In this tutorial article, we first explain the three major ordinal model classes; the cumulative, sequential and adjacent category models. We then show how to fit ordinal models in a fully Bayesian framework with the $\mathrm{R}$ package brms, using data sets on stem cell opinions and marriage time courses. Appendices provide detailed mathematical derivations of the models and a discussion of censored ordinal models. Ordinal models provide better theoretical interpretation and numerical inference from ordinal data, and we recommend their widespread adoption in Psychology.
\end{abstract}

Keywords: ordinal models, Likert items, brms, $\mathrm{R}$

\section{Introduction}

Whenever a variable's categories have a natural order, researchers speak of an ordinal variable (Stevens, 1946). For example, peoples' opinions are often probed with items where respondents choose one of the following response options: "Completely disagree", "Moderately disagree", "Moderately agree", or "Completely agree". Such ordinal data are ubiquitous in Psychology. Although it is widely recognized that such ordinal data are not metric, it is commonplace to analyze them with methods that assume metric responses. However, this practice may lead to serious errors in inference (Liddell \& Kruschke, 2017).

Correspondence concerning this article should be addressed to Paul-Christian Bürkner, Department of Psychology, University of Münster, Fliednerstrasse 21, 48149 Münster, Germany. E-mail: paul.buerkner@ gmail.com 
This tutorial article provides a practical and straightforward solution to the perennial issue of analyzing ordinal variables with the false assumption of metric data: Flexible and easy-to-use Bayesian ordinal regression models implemented in the $\mathrm{R}$ statistical computing environment.

What, specifically, is wrong with analysing ordinal data as if they were metric? This issue was examined in detail by Liddell and Kruschke (2017), whose arguments we summarize here. First, analysing ordinal data with statistical models that assume metric variables, such as $t$-tests and ANOVA, can lead to low correct detection rates, distorted effect size estimates, inflated false alarm (type-I-error) rates, and even inversions of differences between groups. There are three main reasons for these problems. Most importantly, the response categories of an ordinal variable may not be equidistant - an assumption that is required in statistical models of metric responses - but instead the psychological distance between a pair of response options may not be the same for all pairs of response options. For example, the difference between "Completely disagree" and "Moderately disagree" may be much smaller in a survey respondent's mind than the difference between "Moderately disagree" and "Moderately agree".

Second, the distribution of the ordinal responses may be non-normal, in particular if very low or high values are frequently chosen. Third, variances of the unobserved variables that underlie the observed ordinal variables may differ between groups, conditions, timepoints, etc. Such unequal variances cannot be accounted for-or even detected, in some cases - with the ordinal-as-metric approach. Although widely known, these potential pitfalls are ignored whenever a metric model is applied to ordinal data. One common way to address them has been to take averages over several Likert-items, and hope that this averaging makes the problems go away. Unfortunately, that is not the case. Because metric models fail to take into account, or sometimes even detect, these issues, we recommend adopting ordinal models instead: "Often the only method to determine potential problems in an ordinal-as-metric approach is to apply an ordinal model, in which case the results of the ordinal analysis ought to be utilized regardless" (Liddell \& Kruschke, 2017, p. 37).

Historically, appropriate methods for analysing ordinal data were limited, although simple analyses, such as comparing two groups, could be performed with non-parametric approaches (Gibbons \& Chakraborti, 2011). For more general analyses-regression-like methods, in particular - there were few alternatives to incorrectly treating ordinal data as either metric or nominal. However, using a metric or nominal model with ordinal data leads to over- or under-estimating (respectively) the information provided by the data. Fortunately, recent advances in statistics and statistical software have provided many options for approriate models of ordinal response variables. These methods are often summarized under the term ordinal regression models. Nevertheless, application of these methods remains limited, while the use of less appropriate metric models is widespread (Liddell \& Kruschke, 2017).

Several reasons may underlie the persistence with metric models for ordinal data: Researchers might not be aware of more appropriate methods, or they may hesitate to use them because of the perceived complexity in applying or interpreting them. Moreover, since closely related (or even the same) ordinal models are referred to with different names in different contexts, it may be difficult for researchers to decide which model is most relevant 
for their data and theoretical questions. Finally, researchers may also feel compelled to use "standard" analyses because journal editors and reviewers may be sceptical of any "non-standard" approaches. Therefore, there is need for a review and practical tutorial of ordinal models to facilitate their use in psychological research. This tutorial article provides just that.

The structure of this paper is as follows. In Section 2, we introduce three common ordinal model classes. Section 3 is a practical tutorial on fitting ordinal models with two real-world data sets using the $\mathrm{R}$ statistical computing environment ( $\mathrm{R}$ Core Team, 2017). In Section 4, we further motivate the use of ordinal models, and provide practical guidelines on selecting the appropriate model for different research questions and data sets. In two appendices, we provide detailed mathematical derivations and theoretical interpretations of the ordinal models, and an extension of ordinal models to censored data. We hope that the novel examples, derivations, unifying notation, and software implementation will allow readers to better address their research questions regarding ordinal data.

\section{Ordinal model classes}

A large number of parametric ordinal models can be found in the literature. Confusingly, they all have their own names, and their interrelations are often unclear. Fortunately, the vast majority of these models can be expressed within a framework of three distinct model classes (Mellenbergh, 1995; Molenaar, 1983; Van Der Ark, 2001). These are the Cumulative Model, the Sequential Model, and the Adjacent Category Model. We begin by explaining the rationale behind these models in sufficient detail to allow researchers to use them and decide which model best fits their research question and data. Detailed mathematical derivations and discussions are provided in Appendix A.

\subsection{Cumulative model}

For concreteness, we introduce the cumulative model (CM) in the context of an example dataset of opinions about funding stem cell research. The dataset is part of the 2006 US General Social Survey (http://gss.norc.org/) and contains, in addition to opinion ratings, a variable indicating the fundamentalism / liberalism of the respondents' religious beliefs (Agresti, 2010). As an example, we investigate to what extent religious belief predicts opinions about funding stem cell research: Opinion about funding stem cell research is the ordinal dependent variable. The four levels of the Likert item are "definitely not fund" (1), "probably not fund" (2), "probably fund" (3), and "definitely fund" (4). ${ }^{1}$ This is an ordinal variable: The categories have an ordering, but the psychological distance between the categories is not known, nor if the distances are the same across participants. The assumptions of linear models are violated because the dependent variable cannot be assumed to be continuous or normally distributed. Therefore, we are motivated to apply an ordinal model to these data, which are summarized in Table 1.

\footnotetext{
${ }^{1}$ The original ratings were provided in a reverse numerical order, but we reversed the order to allow a more straightforward interpretation where greater values map to more positive constructs.
} 
Table 1

Frequencies of opinion ratings about funding stem cell research

\begin{tabular}{lcccc}
\hline & 1 & 2 & 3 & 4 \\
\hline fundamentalist & 40 & 54 & 119 & 55 \\
moderate & 25 & 41 & 135 & 71 \\
liberal & 23 & 31 & 113 & 122 \\
\hline
\end{tabular}

The CM assumes that the observed ordinal variable $Y$, the opinion rating, originates from the categorization of a latent (not observable) continuous variable $\tilde{Y}$. In this example, $\tilde{Y}$ is the latent opinion about funding stem cell research. To model this categorization process, the CM assumes that there are $K$ thresholds $\tau_{k}$ which partition $\tilde{Y}$ into $K+1$ observable, ordered categories of $Y$. In this example, there are $K+1=4$ response categories, and therefore $K=3$ thresholds. If we assume $\tilde{Y}$ to have a certain distribution (e.g., a normal distribution) with cumulative distribution function $F$, we can write down the probability of $Y$ being equal to category $k$ via

$$
\operatorname{Pr}(Y=k)=F\left(\tau_{k}\right)-F\left(\tau_{k-1}\right)
$$

A conceptual illustration of this idea is shown in the top panel of Figure 1. To make this more concrete, suppose we are interested in the probability of category $k=2$ ("probably not fund") and have $\tau_{1}=-1$ as well as $\tau_{2}=1$. Further, we assume $\tilde{Y}$ to be normally distributed with standard deviation fixed to one and call the corresponding cumulative normal distribution function $\Phi$ (see Figure 4 in Appendix A for a visualization and comparison to other common functions). Then, we compute

$$
\operatorname{Pr}(Y=2)=\Phi\left(\tau_{2}\right)-\Phi\left(\tau_{1}\right)=\Phi(1)-\Phi(-1)=0.84-0.16=0.68 .
$$

However, the above equation does not yet describe a regression model, because there are no predictor variables. We therefore formulate a linear regression for $\tilde{Y}$ with predictor term $\eta=b_{1} x_{1}+b_{2} x_{2}+\ldots$, so that $\tilde{Y}=\eta+\varepsilon$ where $\varepsilon$ describes the error term of the regression. Consequently, $\tilde{Y}$ is split into two parts. The first one $(\eta)$ represents variation in $\tilde{Y}$ that can be explained by the predictors, the second one $(\varepsilon)$ represents variation that remains unexplained. Note that there is no intercept in the predictor term, because the thresholds $\tau_{k}$ replace the model's intercept as both are not identified at the same time. Thus, the CM models the probabilities of $Y$ being equal to category $k$ given the linear predictor $\eta$ via

$$
\operatorname{Pr}(Y=k \mid \eta)=F\left(\tau_{k}-\eta\right)-F\left(\tau_{k-1}-\eta\right) .
$$

We provide a more detailed description and derivation of the model in Appendix A.

The categorization interpretation is natural for many Likert-item data sets, where ordered verbal (or numerical) labels are used to get discrete responses about a possibly continuous psychological variable. Due to the widespread use of Likert-items in Psychology, the CM is possibly the most important ordinal model class for psychological research. It 

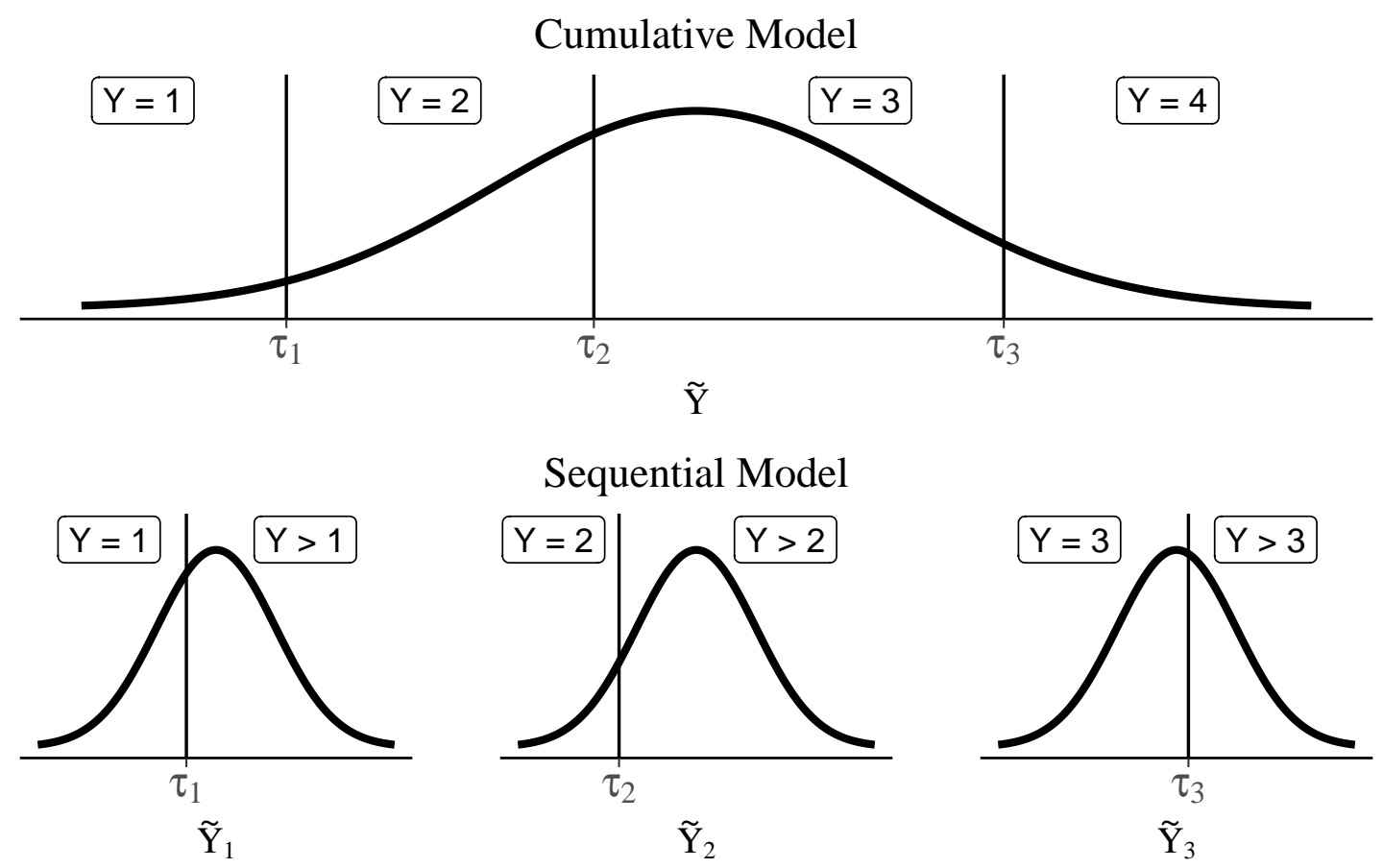

Sequential Model
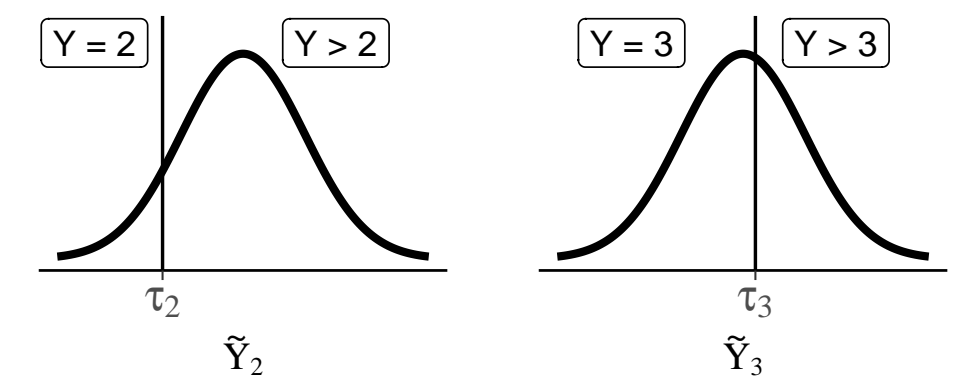

Adjacent Category Model
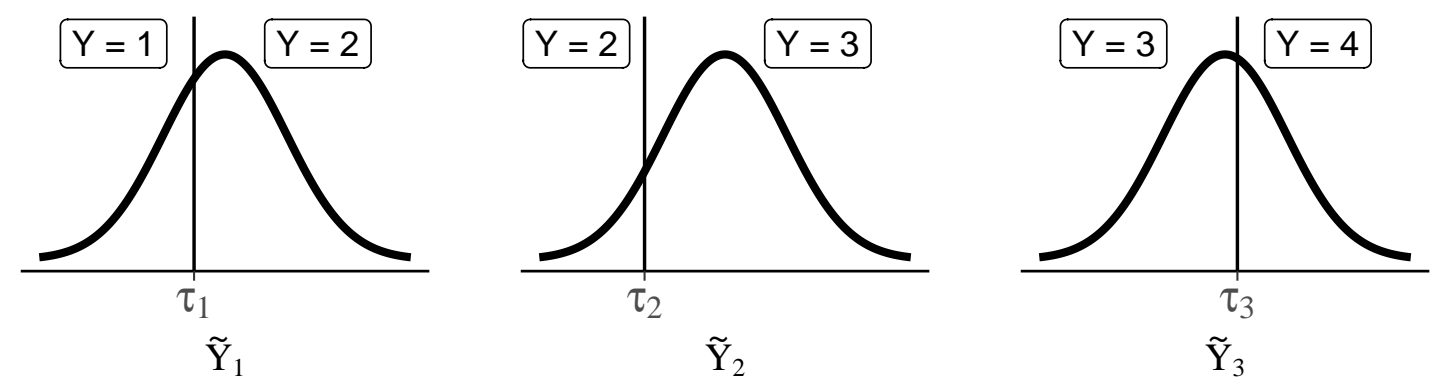

Figure 1. Assumptions of the ordinal model classes. The area under the curve in each bin represents the probability of the corresponding event given the set of possible events for this latent variable. More details are provided in Section 2 and Appendix A. 


\footnotetext{
${ }^{2}$ In linear regression, describing the response as normally distributed around the linear predictor (i.e., the regression line) is equivalent to describing the errors to be normally distributed around zero. The same principle applies to the latent variables in an ordinal model.
}

is reasonable to assume that the stem cell opinion ratings result from categorization of a latent continuous variable - the individual's opinion about stem cell research. Therefore, the cumulative model is theoretically motivated and justified for the example data.

In this example, we wished to predict funding opinion $\tilde{Y}$ from religious belief, which has categories "moderate", "liberal", and "fundamentalist". In the regression model, we use dummy coding with reference category "moderate". Thus, we have two numeric predictor variables $x_{1}$ and $x_{2}$, and the corresponding regression coefficients $b_{1}$ and $b_{2}$ have the following interpretation: $b_{1}$ is the contrast between moderate and liberal, and $b_{2}$ the contrast between moderate and fundamentalist religious belief. The regression model of the individuals' latent opinion about stem cell research is thus

$$
\tilde{Y}=\eta+\varepsilon=b_{1} x_{1}+b_{2} x_{2}+\varepsilon
$$

We assume the latent variable $\tilde{Y}$ (or equivalently the error term $\varepsilon$ ) to be normally distributed $^{2}$ with standard deviation fixed to one. As above, we call the corresponding cumulative normal distribution function $\Phi$. Then, the probabilities for each response category $k$ can be computed as follows:

$$
\operatorname{Pr}(Y=k)=\Phi\left(\tau_{k}-\left(b_{1} x_{1}+b_{2} x_{2}\right)\right)-\Phi\left(\tau_{k-1}-\left(b_{1} x_{1}+b_{2} x_{2}\right)\right) .
$$

The parameters to be estimated are the three thresholds $\tau_{1}$ to $\tau_{3}$ as well as the two regression coefficients $b_{1}$ and $b_{2}$. In Section 3.1, we show how to fit this model in the $\mathrm{R}$ programming language environment.

\subsection{Sequential model}

We introduce the sequential model (SM) in the context of an example real-life data set concerning marriage duration. The data are from the US National Survey of Family Growth 2013 - 2015 (NSFG; https://www.cdc.gov/nchs/nsfg), in which data were gathered about family life for over 10,000 individuals. We will focus on a sample of 1597 women, who had been married at least once in their life at the time of the survey. Inspired by Teachman (2011), who used the NSFG 1995 data, we are interested in predicting the duration, in years, of first marriage. For now, we only consider divorced couples in order to illustrate the main ideas of the sequential model. If we included non-divorced women in the data, the data would be called censored because the event (divorce) was not observed. Although modeling censored data is possible in the SM, we defer this additional complexity to Appendix B. The first ten rows of the data are shown in Table 2.

For many ordinal variables, the assumption of a single underlying continuous variable may not be appropriate. If the response can be understood as being the result of a sequential process, such that a higher response category is possible only after all lower categories are achieved, the sequential model as proposed by Tutz (1990) is usually appropriate. For 
Table 2

Overview of marriage data from the

NSFG 2013-2015 survey.

\begin{tabular}{lllll}
\hline ID & together & age & years & divorced \\
\hline 1 & yes & 19 & 9 & TRUE \\
2 & yes & 22 & 9 & FALSE \\
3 & yes & 20 & 5 & FALSE \\
4 & yes & 22 & 2 & FALSE \\
5 & yes & 25 & 6 & FALSE \\
6 & yes & 30 & 1 & FALSE \\
7 & yes & 32 & 9 & FALSE \\
8 & no & 24 & 14 & TRUE \\
9 & no & 37 & 1 & TRUE \\
10 & yes & 18 & 13 & TRUE \\
\hline
\end{tabular}

Note. In the main analysis, only data of divorced women were used.

example, a couple can divorce in the 7th year only if they haven't already divorced in their first six years of marriage: Duration of marriage in years - the ordinal dependent variable $Y$ in the current example - can be thought of as resulting from a sequential process.

The SM assumes that for every category $k$-year of marriage in our example - there is a latent continuous variable $\tilde{Y}_{k}$ that determines the transition between the $k$ th and the $k+1$ th category. In the marriage example, $\tilde{Y}_{k}$ represents all the factors contributing to the probability of a couple's marriage continuing beyond a given year $k$. Informally, we could call $\tilde{Y}_{k}$ "marriage quality", in the ongoing example. The categories are separated by thresholds $\tau_{k}$-perhaps thought of as the combination of all factors working against the marriage continuing beyond year $k$ in our example. If $\tilde{Y}_{k}$ is greater than the threshold $\tau_{k}$, the sequential process - e.g. marriage - continues; otherwise it stops at category $k$. The SM is illustrated in the middle panel of Figure 1.

Since the thresholds $\tau_{k}$ refer to different latent variables, they don't need to be ordered. That is, we may as well have $\tau_{k+1}<\tau_{k}$. Similar to what we did in the derivation of the CM, we need to assume a certain distribution for $\tilde{Y}_{k}$ (e.g., a normal distribution) with cumulative distribution function $F$. Let's suppose, we want to model the probability of divorce in the third year. This means that divorce did not happen in the first year $\left(\tilde{Y}_{1}>\tau_{1}\right)$, it did not happen in the second year $\left(\tilde{Y}_{2}>\tau_{2}\right)$, but that it happened in the third year $\left(\tilde{Y}_{3} \leq \tau_{3}\right)$. We can write this as follows:

$$
\begin{aligned}
P(Y=3) & =P\left(\tilde{Y}_{1}>\tau_{1}\right) P\left(\tilde{Y}_{2}>\tau_{2}\right) P\left(\tilde{Y}_{3} \leq \tau_{3}\right) \\
& =\left(1-P\left(\tilde{Y}_{1} \leq \tau_{1}\right)\right)\left(1-P\left(\tilde{Y}_{2} \leq \tau_{2}\right)\right) P\left(\tilde{Y}_{3} \leq \tau_{3}\right)
\end{aligned}
$$

If we further assume $Y_{1}, Y_{2}$, and $Y_{3}$ to be standard normally distributed and set, 
just for illustration purposes, $\tau_{1}=0, \tau_{2}=-1$ and $\tau_{3}=1$ we can explicitely compute the probability of divorce in the third year:

$$
P(Y=3)=\left(1-\Phi\left(\tau_{1}\right)\right)\left(1-\Phi\left(\tau_{2}\right)\right) \Phi\left(\tau_{3}\right)=(1-\Phi(0))(1-\Phi(-1)) \Phi(1)=0.35
$$

To make the SM an actual regression model, we set up a linear regression for each latent variable via $\tilde{Y}_{k}=\eta+\varepsilon_{k}$ with a category specific error term $\varepsilon_{k}$. By default, all $\tilde{Y}_{k}$ share the same linear predictor $\eta$, such that the effect of any potential predictor is constant across $k$. (Say, age at marriage is related to $\tilde{Y}_{k}$ identically for years $k=3$ and $k=9$.) This implies the following probability for the category $k$, or duration of marriage, under the sequential model:

$$
\operatorname{Pr}(Y=k \mid \eta)=F\left(\tau_{k}-\eta\right) \prod_{j=1}^{k-1}\left(1-F\left(\tau_{j}-\eta\right)\right)
$$

In words, the probability that $Y$ falls in category $k$ is equal to the probability that it did not fall in one of the former categories 1 to $k-1$, and-when it comes to the decision whether to stop at $k$ or continue beyond it - the process stopped. In the current example, we will use the survey respondents' age at marriage and whether the couple was already living together before marriage as predictors of marriage duration. We can think of the years of marriage as a sequential process: Each year, the marriage may continue or end by divorce, but the latter can only happen if it did not happen before. The years of marriage until divorce is our response variable $Y$, whereas age at marriage and whether the couple was already living together before marriage are our predictor variables, which we denote as $x_{1}$ and $x_{2}$, respectively. As the latter predictor is categorical, it will be dummy coded for our analysis with $x_{2}=1$ if the couple was already living together and $x_{2}=0$ otherwise. This implies the following linear regression for the latent variables $\tilde{Y}_{k}$ :

$$
\tilde{Y}_{k}=b_{1} x_{1}+b_{2} x_{2}+\varepsilon_{k}
$$

We assume an extreme-value distribution for $\tilde{Y}_{k}(F=\mathrm{EV})$, because it is the most common choice in discrete time-to-event / survival models. This function is graphically compared to other alternatives in Figure 4 in Appendix A. Together, this implies that the probability of a marriage ending in the $k$ th year can be computed as follows:

$$
\operatorname{Pr}(Y=k)=\operatorname{EV}\left(\tau_{k}-\left(b_{1} x_{1}+b_{2} x_{2}\right)\right) \prod_{j=1}^{k-1}\left(1-\operatorname{EV}\left(\tau_{j}-\left(b_{1} x_{1}+b_{2} x_{2}\right)\right)\right)
$$

For the current data set, the last marriage was divorced after 27 years and so we have 26 thresholds $\left(\tau_{1}\right.$ to $\left.\tau_{26}\right)$ to estimate in addition to the two regression coefficients $b_{1}$ and $b_{2}$. In Section 3.2, we will learn how to fit this model in the $\mathrm{R}$ programming language environment. 


\subsection{Adjacent category model}

The adjacent category model (ACM) is a widely used ordinal model in item-response theory and is applied in many large scale assessment studies such as PISA (OECD, 2017). It is somewhat different to the CM and SM because it is difficult to think of a natural process leading to it. Therefore, the ACM can be chosen for its mathematical convenience rather than any quality of interpretation. Consequently, we do not include an example specifically dedicated to the ACM, but will illustrate its use when we fit ordinal models to the stem cell data set. In the ACM, we predict the decision between two adjacent categories $k$ and $k+1$ using latent variables $\tilde{Y}_{k}$, with thresholds $\tau_{k}$ and cumulative distribution function $F$. If $\tilde{Y}_{k}<\tau_{k}$ we choose category $k$, else we choose category $k+1$. The decision process assumed by the ACM is illustrated in the bottom panel of Figure 1. We can formally write this as follows:

$$
P(Y=k \mid Y \in\{k, k+1\})=F\left(\tau_{k}\right)
$$

This is superficially similar to the SM, but with an important disctintion. SM models the decision between $Y=k$ and $Y>k$, while the ACM models the decision between $Y=k$ and $Y=k+1$. To make the latter more concrete, suppose that the latent variable $\tilde{Y}_{2}$ is standard normally distributed (with distribution function $\Phi$ ) and $\tau_{2}=1$, then the probability of choosing $Y=2$ ("probably not fund") over $Y=3$ ("probably fund") in the stem cell example would be

$$
P(Y=2 \mid Y \in\{2,3\})=\Phi\left(\tau_{2}\right)=\Phi(1)=0.84
$$

Including the linear predictor $\eta$ into this model leads to the general equation

$$
P(Y=k \mid Y \in\{k, k+1\}, \eta)=F\left(\tau_{k}-\eta\right) .
$$

Under the ACM, the (unconditional) probability of the response $Y$ being equal to category $k$ given $\eta$ (i.e., $P(Y=k \mid \eta)$ ) is computed with a quite extensive formula shown in Appendix A.

\subsection{Generalizations of ordinal models}

We have introduced the three most important ordinal model classes, and refer readers to Appendix A for more details on each of them. An overview of the three model classes, and how to apply them with the software package described below, is shown in Box 1. However, before proceeding to fitting ordinal models in $\mathrm{R}$, we briefly consider two generalizations of the models discussed above; category-specific effects and unequal variances. 


\section{Box 1. Overview of ordinal models and brms syntax.}

Consider an observed ordinal response variable $Y$, and a predictor $X$. The three model classes can be summarized as follows:

1. Cumulative model $(\mathrm{CM})$

- $Y$ originates from categorization of a latent variable $\tilde{Y}$.

- $\operatorname{brm}(\mathrm{Y} \sim \mathrm{X}$, family $=\operatorname{cumulative}(), \ldots)$

- Example: A 5-point Likert item response predicted from gender.

2. Sequential model (SM)

- $Y$ is result of a sequential process.

- $\operatorname{brm}(\mathrm{Y} \sim \mathrm{X}$, family $=\operatorname{sratio}(), \ldots)$

- Example: Number of cars bought predicted from age.

3. Adjacent category model (ACM)

- Model the decision between two adjacent categories of $\tilde{Y}$.

- $\operatorname{brm}(\mathrm{Y} \sim \mathrm{X}$, family $=\operatorname{acat}(), \ldots)$

- Example: Number of correctly solved sub-items of a complex math task.

Generalizations of ordinal models include:

1. Category specific effects

- Can be modeled within ACM and SM.

- $\operatorname{brm}(\mathrm{Y} \sim \operatorname{cs}(\mathrm{X})$, family $=\operatorname{acat}() / \operatorname{sratio}(), \ldots)$

- Example: Likert item responses predicted from gender, such that gender is expected to affect high responses differently than low responses.

2. Unequal variances

- Can be modeled within all three ordinal model classes.

- $\operatorname{brm}(b f(Y \sim X, \operatorname{disc} \sim \mathrm{X}), \ldots)$

- Example: Likert item responses predicted from gender, where the variances of the latent variables differ between genders.

Note: ... indicates additional arguments to brm(), such as specifying a data set.

2.4.1 Category-specific effects. In all of the ordinal models thusfar, all predictors are by default assumed to have the same effect on all response categories, which may not always be an appropriate assumption. It is often possible that a predictor has different impacts for different response categories of $Y$. For example, religious belief may have little relation to whether people endorse a "definitely not fund" (1) over a "probably not fund" (2) opinion about stem cell research, but strongly predict whether "probably fund" (3) is prefered over "definitely fund" (4). In such a case, one can model predictors as having category specific effects so that not one but $K$ coefficients are estimated for this predictor. Doing so is unproblematic in the SM and ACM, but may lead to negative probabilities in the CM and thus problems in the model fitting (see Appendix A). We will come back to this issue below.

2.4.2 Unequal variances. Another generalization of the above models concerns the response function $F$. Especially in the context of CM, $F$ is usually assumed to be a standard normal distribution, that is to have a variance of $v=1$ for reasons of model 
identification. Freely varying the variance $v$ is not possible in ordinal models if all the thresholds $\tau$ are allowed to vary as well. However, it is possible that $v$ varies as a function of group, condition, time, or any other predictor variable provided that the baseline variance is fixed to some value. In other words, $\tilde{Y}$ may have unequal variances across groups, conditions, etc. Ignoring this possibility can lead to problems such as inflated error rates and distorted effect sizes (Liddell \& Kruschke, 2017). Fortunately, unequal variances are easily incorporated in the ordinal models, as we will show below.

\section{$3 \quad$ Fitting ordinal models in $\mathbf{R}$}

Although there are a number of software packages in the $\mathrm{R}$ statistical programming environment ( $\mathrm{R}$ Core Team, 2017) that allow modelling ordinal responses, here we will use the brms (Bayesian Regression Models using Stan) package (Bürkner, 2017, 2018; Carpenter et al., 2017) for several reasons. First, it can estimate all three ordinal model classes introduced above in combination with multilevel structures, category specific effects (except for the cumulative model), unequal variances, and more. Second, brms estimates the models in a Bayesian framework, which provides considerably more information about the model and its parameters (Gelman et al., 2013; McElreath, 2016), allows a more natural quantification of uncertainty (Kruschke, 2014), and is able to estimate models for which traditional maximum likelihood based methods fail (Eager \& Roy, 2017). A brief description of the basic concepts of Bayesian statistics is provided in Box 2 (see also Kruschke \& Liddell, 2018a, 2018b). For a general introduction to brms see Bürkner (2017) and Bürkner (2018). We provide brief notes on ordinal models using other software packages in Section 4 .

In the tutorial below, we assume that readers know how to load data sets into $\mathrm{R}$, and execute other basic commands. Readers unfamiliar with $\mathrm{R}$ may consult free online $\mathrm{R}$ tutorials $^{3}$. The complete $\mathrm{R}$ code for this tutorial, including the example data used here, can be found at (https://osf.io/cu8jv/). To follow the tutorial, users first need to install the required brms $\mathrm{R}$ package. Packages should only be installed once, and therefore the following code snippet should only be run once:

install.packages ("brms")

Then, in order to have the brms functions available in the current $\mathrm{R}$ session, users must load the package at the beginning of every session:

library (brms)

Next, we present two real-world data sets from different areas of psychology that contain ordinal variables as the main dependent variable. We remind the readers that ordinal data is not limited to the types of variables introduced here, but can be found in a wide variety of research areas, as noted by Stevens (1946): "As a matter of fact, most of the scales used widely and effectively by psychologists are ordinal scales" (p.679).

\footnotetext{
${ }^{3} \mathrm{~A}$ brief introduction to $\mathrm{R}$ basics can be found at http://blog.efpsa.org/2016/12/05/ introduction-to-data-analysis-using-r/ (Vuorre, 2016). For a comprehensive, book-length tutorial, we recommend https://r4ds.had.co.nz (Wickham \& Grolemund, 2016).
} 


\section{Box 2. Basics of Bayesian Statistics.}

Bayesian statistics focuses on the posterior distribution $p(\theta \mid Y)$ where $\theta$ are the model parameters (unknown quantities) and $Y$ are the data (known quantities) to condition on. The posterior distribution is generally computed as

$$
p(\theta \mid Y)=\frac{p(Y \mid \theta) p(\theta)}{p(Y)} .
$$

In the above equation $p(Y \mid \theta)$ is the likelihood, $p(\theta)$ is the prior distribution and $p(Y)$ is the marginal likelihood. The likelihood $p(Y \mid \theta)$ is the distribution of the data given the parameters and thus relates the the data to the parameters. The prior distribution $p(\theta)$ describes the uncertainty in the parameters before having seen the data. It thus allows to explicitely incorporate prior knowledge into the model. The marginal likelihood $p(Y)$ serves as a normalizing constant so that the posterior is an actual probability distribution. Except in the context of specific methods (i.e., Bayes factors), $p(Y)$ is rarely of direct interest.

In classical frequentist statistics, parameter estimates are obtained by finding those parameter values that maximise the likelihood. In contrast, Bayesian statistics estimate the full (joint) posterior distribution of the parameters. This is not only fully consistent with probability theory, but also much more informative than a single point estimate (and an approximate measure of uncertainty commonly known as 'standard error'). Obtaining the posterior distribution analytically is rarely possible and thus Bayesian statistics relies on Markov-Chain Monte-Carlo (MCMC) methods to obtain samples (i.e., random values) from the posterior distribution. Such sampling algorithms are computationally very intensive and thus fitting models using Bayesian statistics is usually much slower than in frequentist statistics. However. advantages of Bayesian statistics - such as greater modeling flexibility, prior distributions, and more informative results - are often worth the increased computational cost.

\subsection{Opinion about funding stem cell research}

First, we will analyse the stem cell data set introduced above (see Table 1). We wish to predict the respondents' opinion about funding stem cell research (variable rating in Table 1) from the degree of fundamentalism of their religious beliefs (variable belief). This model can easily be fitted using the brm() function by providing it three arguments, as shown below:

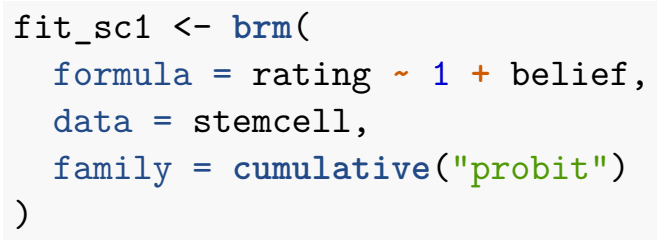

The three arguments inside brm() were, formula, data, and family, respectively. First, and perhaps most important, the formula argument identifies which variable(s) is the dependent variable, and which variable(s) the predictor variable. The model formula is specified with standard $\mathrm{R}$ modeling syntax, where dependent variables are written on the left-hand side of $\sim$ and the predictors on the right-hand side, separated with + s. Interactions between predictors, if desired, are specified by separating them with $*$ instead of + . The 1 
on the right-hand side of $\sim$ means that an intercept (i.e. the thresholds in ordinal models) should be included. Although it is included automatically, we added it here for clarity. Note also that $\mathrm{R}$ functions allow the arguments to be specified in order, such that if the expected order is known, the argument doesn't have to be named.

In addition, we provided the data and the family arguments. The former takes a data frame from the current $\mathrm{R}$ environment. The latter defines the distribution of the response variable, i.e. the specific ordinal model we wish to use, and the desired transformation we want to apply to the predictor term - which is nothing else than the distribution function $F$ in ordinal models. We specified cumulative ("probit") in order to apply a cumulative model assuming the latent variable (or equivalently the error term $\varepsilon$ ) to be normally distributed. If we had omitted "probit" from the specification of the family, the default logistic distribution would have been assumed instead (see Appendix A for details).

The model (which we saved into the fit_sc1 variable) is readily summarized via summary(fit_sc1)

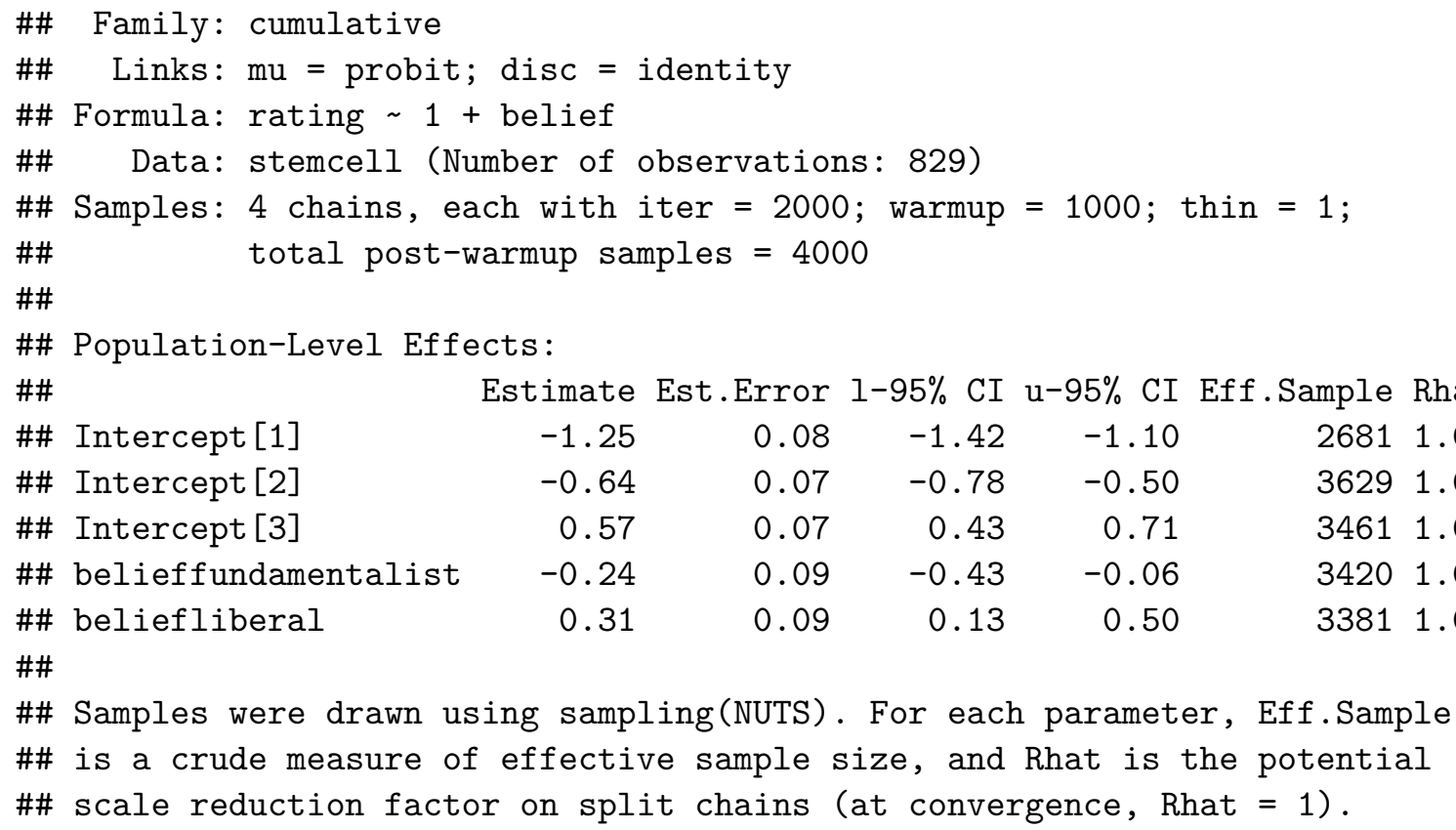

For consistency with other model classes that brms supports, thresholds in ordinal models are called "intercepts" although, from a theoretical perspective, they are not quite the same. In addition to the regression coefficients (which are displayed under the heading Population-Level Effects), this display includes information about the model (first three rows), data, and the Bayesian estimation algorithm (Samples row; e.g., see Bürkner, 2017; van Ravenzwaaij, Cassey, \& Brown, 2016).

Of most importance to us are the regression coefficients. The Estimate column provides the posterior means of the parameters, and Est.Error the parameters' posterior standard deviations. These quantities are analogous, but not identical, to frequentist point estimates and standard errors, respectively. 1-95\% CI and u-95\% CI provide the bounds 
of the $95 \%$ credible intervals (CIs; Bayesian confidence intervals; the numbers refer to the 2.5 and 97.5 percentiles of the posterior distribution). Although credible intervals can be numerically similar to their frequentist counterparts, confidence intervals, they actually lend themselves to an intuitive probabilistic interpretation, unlike the latter which are often mistakenly so interpreted (Hoekstra, Morey, Rouder, \& Wagenmakers, 2014; Morey, Hoekstra, Rouder, Lee, \& Wagenmakers, 2015). To get different CIs, use the prob argument (e.g., summary(fit_sc1, prob $=.99$ ) for a $99 \% \mathrm{CI}$ ).

The two additional columns named Eff.Sample and Rhat, which indicate whether the model fitting algorithm converged to the underlying values, are briefly explained in the last three rows of the output. In short, Rhat should not be larger than 1.1 and Eff. Sample (i.e., "effective sample size") should be as large as possible. For most applications, Eff. Sample $>1000$ is sufficient for stable estimates. Because these quantities are not the focus of this paper - and convergence is not a problem for any of the models considered here - we refer the reader to Bürkner (2017) for more details.

The first three rows of the output under Population-Level Effects describe the three thresholds of the CM as applied to the stem cell funding opinion data. Recall from above that when the cumulative distribution function $F=\Phi$ (standard normal distribution), $\tilde{Y}$ is a standard normal variable. Consequently, the thresholds are standard normal deviates and therefore indicate where the continuous latent variable $\tilde{Y}$ is partitioned to produce the observed responses $Y$, in standard deviation units. Therefore, applying $\Phi$ to each threshold leads to the cumulative probability of responses below that threshold if all predictor variables were zero. Although it is important to be able to interpret the thresholds, similar to ordinary regression intercepts, they are rarely of central focus in the modelling endeavor. Instead, we are most interested in the regression coefficients $b_{1}$ and $b_{2}$, to which we turn next.

Because belief was coded as a factor in $\mathrm{R}$ with moderate as the reference category, the coefficients belieffundamentalist and beliefliberal indicate the extent to which people with fundamentalist and liberal religious beliefs differ from those with moderate beliefs on the latent scale $\tilde{Y}$ of opinion in stem cell funding. The point estimate of beliefliberal indicates that people with liberal beliefs hold 0.31 standard deviations more positive opinions toward funding stem cell research on the latent opinion scale $\tilde{Y}$. The 95\%-CI of this parameter is between 0.13 and 0.50 and so does not include zero. We can therefore conclude with at least $95 \%$ probability that people with liberal religious beliefs hold more positive opinions regarding the funding of stem cell research than do people with moderate religious beliefs.

People with fundamentalist religious beliefs, on the other hand, have more negative opinions regarding funding of stem cell research than do people with moderate religious beliefs. The former's opinions about stem cell research funding are 0.24 standard deviations more negative than those of the latter, on the latent opinion scale. This parameter is between -0.43 and -0.06 with $95 \%$ probability.

The results can also be summarized visually by plotting the estimated relationship between belief and rating. Figure 2 displays the estimated probabilities of the four response categories for the three religious belief groups. It becomes quite clear that fundamentalists 


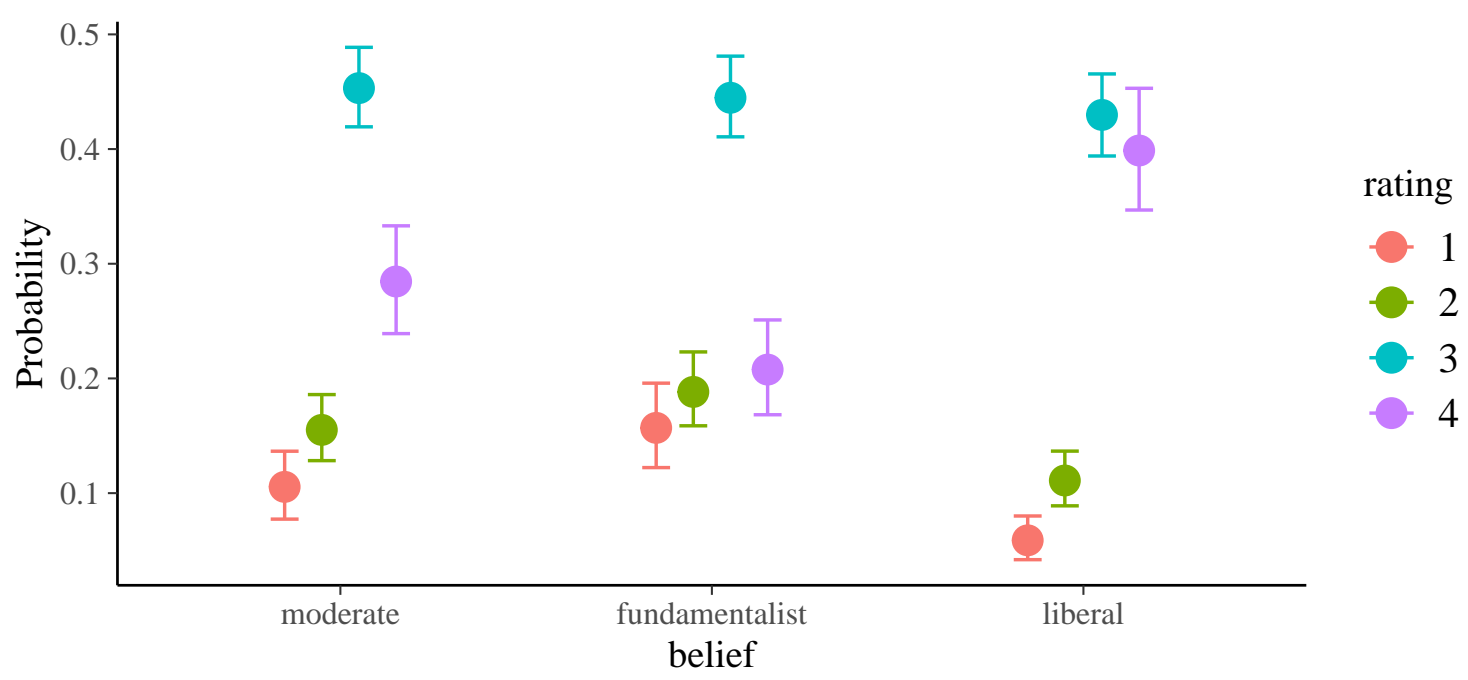

Figure 2. Marginal effects of religious belief on opinion about funding stem cell research based on model fit_sc1. Points indicate the posterior mean estimates of the probability of ratings in each opinion rating category (indicated by color) for each of the three groups (x-axis). Error bars indicate $95 \%$ Credible Intervals.

have stronger opinion against funding stem cell research because they are less likely to respond with "definitely fund" (4) than either of the two groups. Similarly, they are more likely to respond "definitely not fund" (1) and "probably not fund" (2) than the other two groups are. The code to produce this figure is:

marginal_effects(fit_sc1, "belief", categorical = TRUE)

3.1.1 Category-specific effects. Above, we assumed that the effect of religious belief is equal across the opinion rating categories. That is, there was only one predictor term for each of fundamentalist and liberal beliefs' effects on funding opinion. However, this assumption may not be appropriate, and beliefs may impact opinions differently depending on the rating category. For example, it is possible that individuals with liberal beliefs are more likely to rate their funding opinion with the highest rating than are individuals with moderate beliefs, but that the two groups would not otherwise differ in their opinion ratings. When the effects of predictors can vary in this manner across categories, we call the resulting model to have category-specific effects.

Next, we investigate whether belief has category specific effects. In other words, does belief's relationship to funding opinion vary across response categories? However, fitting category specific effects in cumulative models is problematic because of the possibility of negative probabilities and therefore not allowed in brms (see Appendix A). Therefore, we use the adjacent category model instead. To specify an adjacent category model, use family $=$ acat () instead of family = cumulative(), as an argument to the brm() function. Then, to model belief with possible category specific effects, wrap it in cs() in the model's formula, as shown below: 
Table 3

Summary of regression coefficients for the category-specifc adjacent category model fitted to the stemcell data.

\begin{tabular}{llll}
\hline & Estimate & l-95\% CI & $\mathrm{u}-95 \% \mathrm{CI}$ \\
\hline Intercept[1] & -0.32 & -0.62 & 0.01 \\
Intercept[2] & -0.73 & -0.94 & -0.52 \\
Intercept[3] & 0.40 & 0.22 & 0.58 \\
belieffundamentalist[1] & -0.13 & -0.53 & 0.28 \\
belieffundamentalist[2] & -0.24 & -0.54 & 0.04 \\
belieffundamentalist[3] & -0.08 & -0.33 & 0.19 \\
beliefliberal[1] & -0.12 & -0.57 & 0.34 \\
beliefliberal[2] & 0.06 & -0.25 & 0.36 \\
beliefliberal[3] & 0.45 & 0.21 & 0.68 \\
\hline
\end{tabular}

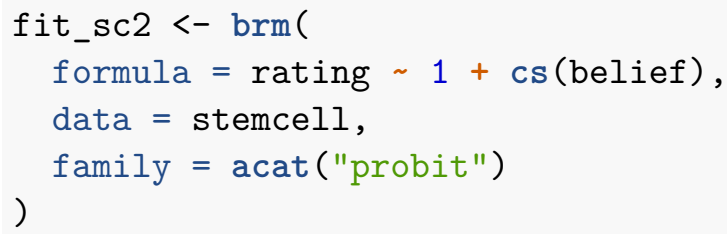

\footnotetext{
${ }^{4}$ The assumption of equal variances of residuals can be relaxed in linear regression models as well. However, with ordinal models, (un)equal variances refer to the latent variable $\tilde{Y}$ and not to the manifest variable $Y$ (Liddell \& Kruschke, 2017).
}

As shown in Table 3, liberals prefer response 4 ("definitely fund") over response 3 ("probably fund") much more than moderates with a coefficient of $\mathrm{b}=0.45(95 \%$-CI $=[0.21$, $0.68]$ ). At the same time, there is little difference between liberals and moderates for the other response categories; parameters beliefliberal[1] and beliefliberal [2] indicate differences between moderates' and liberals' preferences for response 2 over response 1, and response 3 over response 2 , respectively. In contrast, fundamentalists prefer lower response categories than moderates throughout, but the differences are quite small and uncertain - as indicated by the rather wide $95 \%$-CIs that also overlap zero.

It can be more difficult to interpret the sizes of the ACM's coefficients, in contrast to ones from the CM. Thus, to better understand the magnitudes of the effects, we recommend plotting the model's predicted values (for instance, via marginal_effects (fit_sc2)). With these data, the resulting figure looks very similar to Figure 2 and thus we do not show it here.

3.1.2 Unequal variances. As discussed in Section 2.4.2, by default we assume the variance of the latent variable to be the same throughout the model, an assumption unavoidable in linear regression. ${ }^{4}$ Within the framework of ordinal models in brms, we can relax this assumption. For the stem cell data, this implies asking whether variances of the stem cell funding opinion differ across categories of religious belief.

Conceptually, unequal variances are incorporated in the model by specifying an 


\footnotetext{
${ }^{5}$ Notice that the transformation must be done on the posterior samples of disc, not its posterior summary. The $\mathrm{R}$ code to transform disc to $s$ is shown on OSF (https://osf.io/cu8jv/). Please also notice that in the summary output of brms, coefficients of the log-discrimination just have the prefix disc_although they are in fact on the log-scale.
}

additional regression formula for the variance component of the latent variable $\tilde{Y}$. In brms, the parameter related to latent variances is called disc (short for "discrimination"), following conventions in item response theory. Importantly, disc is not the variance itself, but the inverse of the standard deviation, $s$. That is, $s=1 /$ disc. Further, because disc must be strictly positive, it is by default modeled on the log-scale.

Predicting auxiliary parameters (parameters of the distribution other than the mean/location) in brms is accomplished by passing multiple regression formulas to the brm() function. To do so, these formulas must first be wrapped in another function, bf () or If () - depending on whether it is a main or an auxiliary formula. These formulas are then combined and passed to the formula argument of brm(). Because the standard deviation of the latent variable is fixed to one for the baseline group (moderates), disc cannot be estimated for all three groups of religous belief. We must therefore ensure that disc is only estimated for the liberals and fundamentalists. To do so, we omit the intercept from the model of disc by writing $0+\ldots$ on the right-hand side of the regression formula. By default, $\mathrm{R}$ applies cell-mean coding (cmc) to factors in formulas without an intercept. That would lead to disc being estimated for all three groups, so we must deactivate it via the cmc argument of $\mathrm{lf}()$. With this in mind, an unequal variance $\mathrm{CM}$ of the stemcell data is specified as follows:

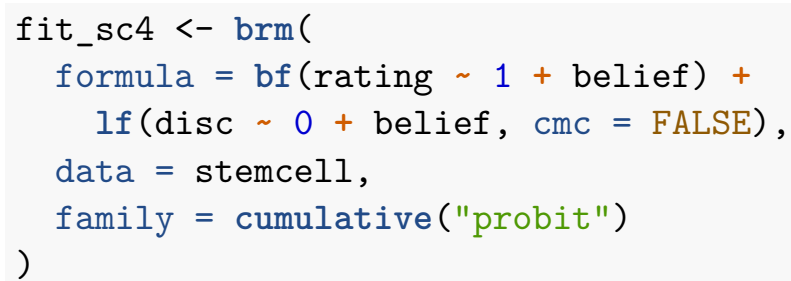

The syntax for specifying an unequal variance is identical to the syntax of an equal variance model with one important addition: A formula for the disc parameter was added, using a + between the formulas. The formula was wrapped in $\mathrm{lf}($ ) ("linear formula") to indicate that an auxiliary parameter, such as disc, is predicted.

The estimated parameters of the unequal variance model are summarized in Table 4. As discussed above, disc is the inverse of the standard deviation of $\tilde{Y}$, and by default modeled through a log-link, that is we predict $\log ($ disc $)$ instead of disc. To also display the standard deviations $s$, we transformed $\log (\operatorname{disc})$ to $s$ with $s=1 / \exp (\log (\operatorname{disc})) .{ }^{5}$ The standard deviation of the latent variable was higher for liberals $(\mathrm{SD}=1.26 ; 95 \%$-CI $=[1.06$, 1.50]) than for moderates for whom the standard deviation was fixed to 1 to identify the model. The standard deviation for fundamentalists $(\mathrm{SD}=1.09 ; 95 \%-\mathrm{CI}=[0.93,1.28]$ ) was also somewhat higher than for moderates although this difference was not substantial, nor did the CI exclude zero. The main regression coefficients of religious belief also changed slightly, however the main result that liberals tend to prefer more positive responses, and fundamentalists tend to prefer more negative categories than moderates, was similar to the 
Table 4

Summary of regression coefficients for the cumulative model with unequal variances fitted to the stemcell data.

\begin{tabular}{llll}
\hline & Estimate & l-95\% CI & u-95\% CI \\
\hline Intercept[1] & -1.36 & -1.56 & -1.17 \\
Intercept[2] & -0.69 & -0.84 & -0.54 \\
Intercept[3] & 0.65 & 0.49 & 0.81 \\
belieffundamentalist & -0.25 & -0.44 & -0.06 \\
beliefliberal & 0.41 & 0.19 & 0.64 \\
log_disc_belieffundamentalist & -0.08 & -0.25 & 0.08 \\
log_disc_beliefliberal & -0.23 & -0.41 & -0.06 \\
sd_belieffundamentalist & 1.09 & 0.93 & 1.28 \\
sd_beliefliberal & 1.26 & 1.06 & 1.50 \\
\hline
\end{tabular}

equal variances model.

3.1.3 Model comparison. We have now fitted three different ordinal models to the stemcell opinion data, and the question naturally arises: Which model should we choose, and base our inference on? For category-specific effects, we saw that many of the resulting coefficients were rather small and uncertain, suggesting that category-specific effects may not be necessary. Similarly, the unequal variance model's parameter estimates suggested that while liberals' opinions might be more variable, those of fundamentalists and moderates were quite similar. One formal approach to model comparison is to investigate the relative fit to data of each of these models. One method to assess this is approximate leave-one-out cross-validation (LOO; Vehtari, Gelman, \& Gabry, 2017), which provides a score that can be interpreted as typical information criteria such as AIC (Akaike, 1998) or WAIC (Watanabe, $2010)^{6}$ in the sense that smaller values indicate better fit. Although a detailed exposition of this topic is beyond the scope of this article, we illustrate how to compare these models' relative fit to the stemcell data using LOO.

First, however, to make sure that differences between fit_sc1 (equal variance CM) and fit_sc2 (ACM with category-specific effects) are not due to using another ordinal model class, we also fit the ACM without category specific effects. The syntax is very similar to shown above, but without cs(); we therefore omit the code here and saved the model in fit_sc3. The comparison between the four ordinal models using approximate leave-one-out cross-validation is done via

10o(fit_sc1, fit_sc2, fit_sc3, fit_sc4)

We then display the estimated model comparison metrics (LOOIC for LOO Information Criterion) in Table 5, along with differences in them between models. As can be seen, the cumulative model (fit_sc1) has a somewhat better fit (smaller LOOIC value) than the two ACMs, although the differences are not very large (up to 1 or 2 times the corresponding standard error). Both adjacent category models show very similar LOOIC values, which

\footnotetext{
${ }^{6} \mathrm{AIC}$ and WAIC can be interpreted as approximations of LOO.
} 
Table 5

LOO values and differences between four ordinal models of the stemcell data.

\begin{tabular}{lll}
\hline Model & LOOIC & \multicolumn{1}{c}{ SE } \\
\hline fit_sc1 & $2,040.61$ & 31.10 \\
fit_sc2 & $2,042.80$ & 31.49 \\
fit_sc3 & $2,043.70$ & 30.89 \\
fit_sc4 & $2,039.04$ & 31.22 \\
fit_sc1 - fit_sc2 & -2.20 & 4.94 \\
fit_sc1 - fit_sc3 & -3.10 & 1.74 \\
fit_sc1 - fit_sc4 & 1.57 & 5.16 \\
fit_sc2 - fit_sc3 & -0.90 & 6.07 \\
fit_sc2 - fit_sc4 & 3.76 & 1.52 \\
fit_sc3 - fit_sc4 & 4.66 & 6.34 \\
\hline
\end{tabular}

Note. fit_sc1 $=$ cumulative model with equal variances; fit_sc $2=$ adjacent category model with equal variances and category specific effects; fit_sc3 = adjacent category model with equal variances; fit_sc4 $=$ cumulative model with unequal variances.

implies that estimating category specific effects does not substantially improve model fit. Similarly, the unequal variance CM resulted in only a slightly smaller LOOIC value than the equal variance CM, suggesting that unequal variances improved model fit slightly, but the difference was not substantial.

In the context of model selection, a LOO difference greater than twice its corresponding standard error can be interpreted as suggesting that the model with a lower LOO value fits the data substantially better, at least when the number of observations is large enough ${ }^{7}$. Based on this logic and the results in Table 5, we might prefer fit_sc1 or fit_sc4 (the equal or unequal variance $\mathrm{CM}$, respectively). However, we remind readers that model selection - based on any metric, be it a p-value, Bayes factor, or information criterion - is a controversial and complex topic, and therefore suggest replacing hard cutoff values with context-dependent and theory-driven reasoning. For the current example, we favor the unequal variance CM not only because of its goodness of fit (according to LOOIC), but also because it is parsimonious and theoretically best justified.

3.1.4 Multiple Likert items. Although outside the scope of this tutorial article, we wish to briefly discuss modeling strategies for data with multiple items per person. The extension is straightforward and can be achieved with hierarchical/multilevel modeling.

\footnotetext{
${ }^{7} \mathrm{LOO}$ values and their differences are approximately normally distributed. Hence, for models based on enough observations, we may construct a frequentist confidence interval around the estimate. For instance, a $95 \%$-CI around $\Delta \mathrm{LOO}$ can be constructed via $[\Delta \mathrm{LOO}-1.96 \times \mathrm{SE}(\Delta \mathrm{LOO}), \Delta \mathrm{LOO}+1.96 \times \mathrm{SE}(\Delta \mathrm{LOO})]$.
} 
In the above example, we only had data for one item per person. However, in many studies the participants provide responses to multiple items. For data with multiple items per person, we can fit a multilevel ordinal model that takes the items and participants into account. This allows incorporating all information in the data into the model, while controlling for dependencies between ratings from the same person and between ratings of the same item. For this purpose, the data needs to be in long format, such that each row is an individual rating, with columns for the value of the rating, and identifiers for the participants and items. Suppose that we had measured opinion about funding stem cell research with multiple items and that we call the identifier columns person and item, respectively. Then, we could write the model formula as follows:

rating 1 + belief + (1|person $)+(1 \mid$ item $)$

The notation ( $1 \mid<$ group $>$ ) (e.g., (1|person) or (1|item)) implies that the intercept (1) varies over the levels of the grouping factor (〈group $\rangle$ ). In ordinal models, we have multiple intercepts (recall that they are called thresholds in ordinal models), and (1|<group $\rangle$ ) allows these thresholds to vary by the same amount across levels of group. To model thresholdspecific variances, we would write (cs(1) | 〈group>). For instance, if we wanted all thresholds to vary differently across items so that each item receives its own set of thresholds, we could have added (cs(1) | item) to the model formula.

In summary, this example illustrated the use of CM (with and without unequal variances) and ACM (with and without category-specific effects) in the context of a Likert item response variable. We illustrated how to fit these four models to data using concise $\mathrm{R}$ syntax, enabled by the brm() function, and how to print, interpret, and visualize the model's estimated parameters. Paired with effective visualization (see Figure 2), the models' results are readily interpretable and rich in information due to fully Bayesian estimation. We also found that, in this example, category-specific effects did not meaningfully improve model fit, and that the CMs proved a better fit than the ACMs. Further, there was a small improvement in model fit of the unequal variances $\mathrm{CM}$ over the equal variances CM.

\subsection{Years until divorce}

In the second example, we will analyse the marriage data set introduced in Section 2.2 and Table 2. We wish to predict the duration (in years) of first marriage (variable years), which ends either by divorce or continues beyond the time of the survey. These data can be understood as discrete time-to-event data, with the event of interest being divorce. As predictors we will use the participants' age at marriage (variable age) and whether the couple was already living together before marriage (variable together).

Years of marriage can be thought of as a sequential process: Each year, the marriage may continue or end by divorce, but the latter can only happen if it did not happen before. This clearly calls for use of the sequential model and we seek to predict the time until divorce (i.e., the time until marriage stops; for alternative formulations see Appendix A). Further, we assume an extreme-value distribution for the latent variables $\tilde{Y}_{k}$ (corresponding to the cloglog link in brms; see Appendix A), because it is the most common choice in discrete 


\footnotetext{
${ }^{8}$ This prior is weakly informative for the present model and variable scales. Be aware that for other models or variable scales, this prior may be more informative.
}

time-to-event / survival models. These data can also be modeled using the cumulative model with specific latent distributions such as the extreme-value or Weibull distribution, but for the purpose of this tutorial we focus on the sequential model.

In this section, we only consider divorced couples in order to illustrate the main ideas of the sequential model as fitted in brms. If we included non-divorced women, the data would be called censored because the event (divorce) was not observed. Although modeling censored sequential models in brms is possible, we defer this additional complexity to Appendix B. The model including data of divored couples only is estimated with the following code:

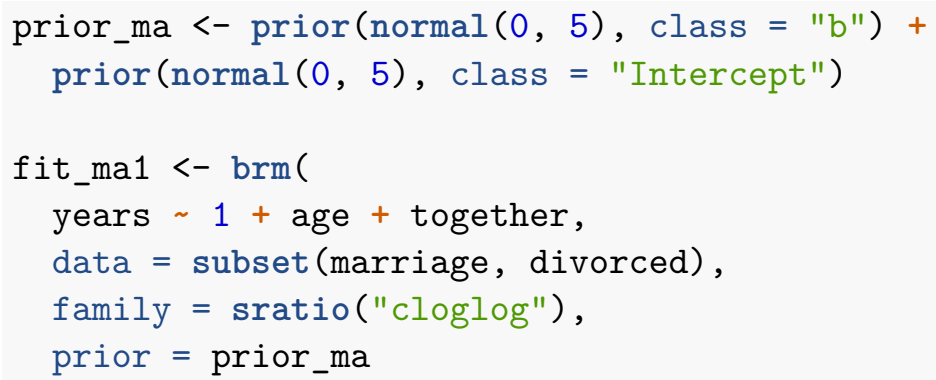

We used weakly informative normal $(0,5)$ priors $^{8}$ for all regression coefficients to improve model convergence, and to illustrate how to specify prior distributions with brms. Trying to fit this model in a frequentist framework would likely lead to serious convergence issues that would be hard to resolve without the ability to specify priors.

After fitting this model, a summary of the results can be displayed with summary (fit_ma1). We find that women who marry later appear to have shorter marriages ( $\mathrm{b}=-0.04 ; 95 \%$-CI $=[-0.07,-0.02] ; 95 \%$-CI exludes zero) while previously living together appears to be unrelated to years of marriage $(b=0.01 ; 95 \%-\mathrm{CI}=[-0.15,0.18])$. As described in Section 2.2, these regression coefficients are defined on the scale of the latent variables $\tilde{Y}_{k}$, which we assumed to be extreme-value distributed. Admittedly, the scale of these coefficients is hard to interpret: The size of the effect $b=-0.04$ of age at marriage is not immediately obvious.

For this reason, we recommend always plotting the results, for instance with marginal_effects(fit_ma1). In this case, years of marriage has a natural metric interpretation. As shown in the left panel of Figure 3, between the minimum and maximum age at marriage (12 and 43 years, respectively) the model predicts a 3.95 year difference in the time until divorce.

However, this model omits an important detail in the data: We only included couples who actually got divorced, and excluded couples who were still married at the end of the study. In the context of time-to-event analysis, this is called (right) censoring, because divorce did not happen up to the point of the end of the study, but may well happen later on in time. Both excluding this information altogether (as we did in the analysis above) or 

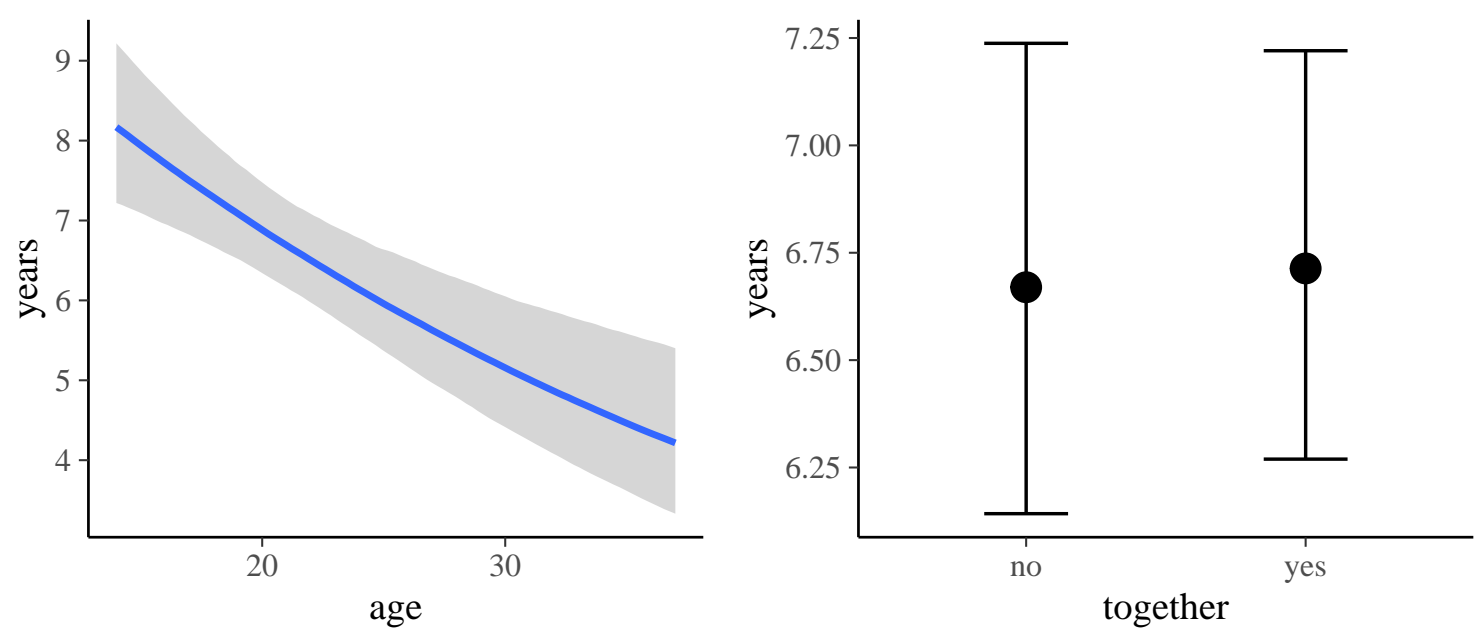

Figure 3. Marginal effects of woman's age at marriage and living together before marriage on the years of marriage until divorce.

falsely treating these couples as having divorced right at the end of the study may lead to bias in the results of unknown direction and magnitude.

For these reasons, we must find a way to incorporate censored data into the model. In the standard version of the sequential model explained in Section 2, each observation must have an associated outcome category. However, for censored data, the outcome category was unobserved. Hence, we will need to expand the standard sequential model, which requires a little bit of extra work, to which we turn to in Appendix B.

\section{Conclusion}

In this tutorial, we introduced three important ordinal model classes both from a theoretical and an applied perspective: The cumulative, sequential, and adjacent category models. The models were formally derived from their underlying assumptions (Appendix A) and applied to real-world data sets covering different psychological fields and research questions. We did not engage in demonstrating (e.g., via simulations) that using ordinal models for ordinal data is superior to other approaches such as linear regression, because this has already been sufficiently covered elsewhere (Liddell \& Kruschke, 2017). Nevertheless, we briefly mention some further arguments in favor of ordinal models.

\subsection{Why should researchers use ordinal regression?}

Although we have highlighted the theoretical justification, and practical ease, of applying ordinal models to ordinal data, one might still object to using these models. We wish to point out here that some of these objections are not sound. First, one might oppose ordinal models on the basis that their results are more difficult to interpret and communicate than those of corresponding linear regressions. The main complexity of ordinal models, in contrast to linear regression, is in the threshold parameters. However, equivalent to 
intercept parameters in linear regression, these parameters rarely are the target of main inference. Usually, researchers are more interested in the predictors, which can be interpreted as ordinary predictors in linear regression models (but keep in mind that they are on the latent metric scale). Furthermore, brms' helper functions make it easy to calculate (see ?fitted.brmsfit) and visualize (?marginal_effects.brmsfit) the model's fitted values (i.e. the predicted marginal proportions for each response category).

Second, it is sometimes the case that one's substantial conclusions do not strongly depend on whether an ordinal or a linear regression model was used. We wish to point out that even though the actionable conclusions may be similar, a linear model will have a lower predictive utility by virtue of assuming a theoretically incorrect outcome distribution. Perhaps more importantly, linear models for ordinal data can lead to effect size estimates that are distorted in size or certainty, and this problem is not solved by averaging multiple ordinal items (Liddell \& Kruschke, 2017).

\subsection{Software options}

Throughout, we have advocated and illustrated the implementation of ordinal models in the $\mathrm{R}$ statistical computing environment using the brms package. The main reason for us advocating these software options is that they are completely free and open source. Therefore, they are available to anyone, without any licensing fees, and are easily extensible. The latter means that many computational and statistical procedures are implemented in $\mathrm{R}$ before they are available in other (commercial) software packages. Further, we believe that the wide variety of models available through the concise and consistent syntax of brms is beneficial to any modeling endeavor (Bürkner, 2017, 2018).

Nevertheless, users may wish to implement ordinal models within their preferred statistical packages. Explaining how to conduct ordinal regressions using other software is outside the scope of this tutorial: Useful references include Heck, Thomas, and Tabata (2013) for IBM SPSS, Bender and Benner (2000) for SAS and S-Plus, and Long, Long, and Freese (2006) for STATA.

\subsection{Choosing between ordinal models}

Equipped with the knowledge about the three ordinal model classes, researchers might still find it difficult to decide which model best fits their research question and data. It is impossible to describe in advance which model would best fit each situation, but we briefly describe some useful rules of thumb for deciding between the models discussed in this paper. An overview of the models is shown in Box 1.

From a theoretical perspective, if the response can be understood as the categorization of a latent continuous construct, we recommend the cumulative model. The categorization interpretation is natural for many Likert-item data sets, where ordered verbal (or numerical) labels are used to get discrete responses about a continuous psychological variable. The cumulative model is also computationally the least intensive, and therefore the fastest model to estimate. If unequal variances are theoretically possible - and they usually are - we 
also recommend incorporating them into the model; ignoring them may lead to increased false alarm rates and inaccurate parameter estimates (Liddell \& Kruschke, 2017). Further, although often overlooked, we think that (differences in) variances can themselves be theoretically interesting, and as such should be modeled.

If the response can be understood as being the result of a sequential process, such that a higher response category is possible only after all lower categories are achieved, we recommend using the sequential model. This model is therefore especially useful, for example, for discrete time data. However, deciding between a categorization and a sequential process may not always be straightforward; in ambiguous situations, estimating both models may be a reasonable strategy.

If category-specific effects are of interest, we recommend using the sequential or adjacent category model. Otherwise, the adjacent category model appears to be more useful than its alternatives only in specific applications (see Appendix A). Category-specific effects are useful when there is reason to expect that a predictor might impact the response variable differently at different levels of the response variable. Finally, we suggest that if one wishes to model ordinal responses, it is important to use any ordinal model instead of falsely assuming metric or nominal responses.

\section{Author Contributions}

PCB generated the idea for the manuscript and wrote the first draft of the theoretical part of the manuscript. Both authors jointly wrote the first draft of the practical part of the manuscript. Both authors critically edited the whole draft and approved the final submitted version of the manuscript. 


\section{References}

Adams, R. J., Wu, M. L., \& Wilson, M. (2012). The Rasch rating model and the disordered threshold controversy. Educational and Psychological Measurement, 72(4), 547-573. doi:10.1177/0013164411432166

Agresti, A. (1984). Analysis of ordinal categorical data. John Wiley \& Sons.

Agresti, A. (2010). Analysis of ordinal categorical data. Chichester: John Wiley \& Sons. doi:10.1002/9780470594001

Akaike, H. (1998). Information theory and an extension of the maximum likelihood principle. In Selected papers of hirotugu akaike (pp. 199-213). Springer.

Ananth, C. V., \& Kleinbaum, D. G. (1997). Regression models for ordinal responses: A review of methods and applications. International Journal of Epidemiology, 26(6), $1323-1333$.

Andersen, E. B. (1973). CONDITIONAL inference for multiple-choice questionnaires. British Journal of Mathematical and Statistical Psychology, 26(1), 31-44. doi:10.1111/j.20448317.1973.tb00504.x

Andersen, E. B. (1977). Sufficient statistics and latent trait models. Psychometrika, 42(1), 69-81.

Andrich, D. (1978a). Application of a psychometric rating model to ordered categories which are scored with successive integers. Applied Psychological Measurement, 2(4), 581-594.

Andrich, D. (1978b). A rating formulation for ordered response categories. Psychometrika, 43(4), 561-573. doi:10.1007/BF02293814

Andrich, D. (2005). The Rasch model explained. In Applied Rasch measurement: A book of exemplars (pp. 27-59). Springer. doi:10.1007/1-4020-3076-2\_3

Bender, R., \& Benner, A. (2000). Calculating Ordinal Regression Models in SAS and S-Plus. Biometrical Journal, 42(6), 677-699. doi:10.1002/1521-4036(200010)42:6<677::AIDBIMJ677>3.0.CO;2-O

Bürkner, P.-C. (2017). brms: An R package for bayesian multilevel models using Stan. Journal of Statistical Software, 80(1), 1-28. doi:10.18637/jss.v080.i01

Bürkner, P.-C. (2018). Advanced bayesian multilevel modeling with the r package brms. The $R$ Journal, 1-15. Retrieved from https://arxiv.org/abs/1705.11123

Carpenter, B., Gelman, A., Hoffman, M. D., Lee, D., Goodrich, B., Betancourt, M., ... Riddell, A. (2017). Stan: A probabilistic programming language. Journal of Statistical Software, $76(1)$.

Cox, D. R. (1972). Regression models and life tables (with discussion). Journal of the Royal Statistical Society, 34, 187-220. 
Cox, D. R. (1992). Regression models and life-tables. In Breakthroughs in statistics (pp. 527-541). Springer.

Eager, C., \& Roy, J. (2017). Mixed Effects Models are Sometimes Terrible. arXiv. Retrieved from http://arxiv.org/abs/1701.04858

Fienberg, S. E. (1980). The analysis of cross-classified categorical data. MIT press.

Fienberg, S. E. (2007). The analysis of cross-classified categorical data. Springer Science \& Business Media.

Fischer, G. H. (1995). The derivation of polytomous Rasch models. In Rasch models (pp. 293-305). Springer. doi:10.1007/978-1-4612-4230-7\_16

Gelman, A., Carlin, J. B., Stern, H. S., Dunson, D. B., Vehtari, A., \& Rubin, D. B. (2013). Bayesian Data Analysis, Third Edition. Boca Raton: Chapman and Hall/CRC.

Gibbons, J. D., \& Chakraborti, S. (2011). Nonparametric statistical inference. In International encyclopedia of statistical science (pp. 977-979). Springer.

Guisan, A., \& Harrell, F. E. (2000). Ordinal response regression models in ecology. Journal of Vegetation Science, 11(5), 617-626.

Heck, R. H., Thomas, S., \& Tabata, L. (2013). Multilevel Modeling of Categorical Outcomes Using IBM SPSS. New York, NY: Routledge.

Hoekstra, R., Morey, R. D., Rouder, J. N., \& Wagenmakers, E.-J. (2014). Robust misinterpretation of confidence intervals. Psychonomic Bulletin $\&$ Review, 21 (5), 1157-1164. doi:10.3758/s13423-013-0572-3

Kruschke, J. K. (2014). Doing Bayesian Data Analysis: A Tutorial Introduction with $R$ (2nd Edition.). Burlington, MA: Academic Press.

Kruschke, J. K., \& Liddell, T. M. (2018a). Bayesian data analysis for newcomers. Psychonomic Bulletin \& Review, 25(1), 155-177.

Kruschke, J. K., \& Liddell, T. M. (2018b). The bayesian new statistics: Hypothesis testing, estimation, meta-analysis, and power analysis from a bayesian perspective. Psychonomic Bulletin \& Review, 25(1), 178-206.

Läärä, E., \& Matthews, J. (1985). The equivalence of two models for ordinal data. Biometrika, 72(1), 206-207.

Liddell, T., \& Kruschke, J. K. (2017). Analyzing ordinal data with metric models: What could possibly go wrong? Journal of Experimental Social Psychology, 79, 328-348.

Linden, W., \& Hambleton, R. K. (1997). Handbook of modern item response theory. New York.

Long, S. J., Long, J. S., \& Freese, J. (2006). Regression Models for Categorical Dependent Variables Using Stata, Second Edition. College Station, TX: Stata Press. 
Masters, G. N. (1982). A Rasch model for partial credit scoring. Psychometrika, 47(2), 149-174. doi:10.1007/BF02296272

McCullagh, P. (1980). Regression models for ordinal data. Journal of the Royal Statistical Society. Series B (Methodological), 109-142.

McElreath, R. (2016). Statistical Rethinking: A Bayesian Course with Examples in $R$ and Stan. CRC Press.

Mellenbergh, G. J. (1995). Conceptual notes on models for discrete polytomous item responses. Applied Psychological Measurement, 19(1), 91-100.

Molenaar, I. (1983). Item steps. Heymans Bulletin HB-83-630-EX). Groningen: University of Groningen, Vakgroep Statistiek En Meettheorie.

Morey, R. D., Hoekstra, R., Rouder, J. N., Lee, M. D., \& Wagenmakers, E.-J. (2015). The Fallacy of Placing Confidence in Confidence Intervals. Psychonomic Bulletin $\&$ Review.

OECD. (2017). PISA 2015: Technical report. Retrieved from http://www.oecd.org/pisa/ data/2015-technical-report/

Peterson, B., \& Harrell, F. E. (1990). Partial proportional odds models for ordinal response variables. Applied Statistics, 205-217.

Rasch, G. (1961). On general laws and the meaning of measurement in psychology. In Proceedings of the fourth berkeley symposium on mathematical statistics and probability (Vol. 4, pp. 321-333). University of California Press Berkeley, CA.

R Core Team. (2017). R: A language and environment for statistical computing. Vienna, Austria: R Foundation for Statistical Computing. Retrieved from https: //www.R-project.org/

Samejima, F. (1969). Estimation of latent ability using a response pattern of graded scores. Psychometrika Monograph Supplement.

Samejima, F. (1972). A general model for free-response data. Psychometrika Monograph Supplement.

Samejima, F. (1995). Acceleration model in the heterogeneous case of the general graded response model. Psychometrika, 60(4), 549-572.

Samejima, F. (1997). Graded response model. In Handbook of modern item response theory (pp. 85-100). Springer.

Stevens, S. S. (1946). On the Theory of Scales of Measurement. Science, 103(2684), 677-680. doi:10.1126/science.103.2684.677

Teachman, J. (2011). Modeling repeatable events using discrete-time data: Predicting marital dissolution. Journal of Marriage and Family, 73(3), 525-540.

Tutz, G. (1990). Sequential item response models with an ordered response. British Journal of Mathematical and Statistical Psychology, 43(1), 39-55. doi:10.1111/j.20448317.1990.tb00925.x 
Tutz, G. (1997). Sequential models for ordered responses. In Handbook of modern item response theory (pp. 139-152). Springer.

Tutz, G. (2000). Die Analyse Kategorialer Daten: Anwendungsorientierte Einführung in Logit-Modellierung und Kategoriale Regression. Oldenbourg: Oldenbourg Verlag.

Van Der Ark, L. A. (2001). Relationships and properties of polytomous item response theory models. Applied Psychological Measurement, 25(3), 273-282. doi:10.1177/01466210122032073

van Ravenzwaaij, D., Cassey, P., \& Brown, S. D. (2016). A simple introduction to Markov Chain Monte-Carlo sampling. Psychonomic Bulletin $\&$ Review, 1-12. doi:10.3758/s13423-016-1015-8

Vehtari, A., Gelman, A., \& Gabry, J. (2017). Practical bayesian model evaluation using leave-one-out cross-validation and waic. Statistics and Computing, 27(5), 1413-1432.

Verhelst, N. D., Glas, C., \& De Vries, H. (1997). A steps model to analyze partial credit. In Handbook of modern item response theory (pp. 123-138). Springer.

Vuorre, M. (2016, December 5). Introduction to Data Analysis using R. JEPS Bulletin. Retrieved December 29, 2016, from http://blog.efpsa.org/2016/12/05/ introduction-to-data-analysis- using-r/

Walker, S. H., \& Duncan, D. B. (1967). Estimation of the probability of an event as a function of several independent variables. Biometrika, 54(1-2), 167-179. doi:10.2307/2333860

Watanabe, S. (2010). Asymptotic equivalence of bayes cross validation and widely applicable information criterion in singular learning theory. Journal of Machine Learning Research, 11 (Dec), 3571-3594.

Wickham, H., \& Grolemund, G. (2016). R for Data Science. Retrieved from http://r4ds. had.co.nz/

Wilson, M. (1992). The ordered artition model: An extension of the partial credit model. Applied Psychological Measurement, 16 (4), 309-325. doi:10.1177/014662169201600401

Wilson, M., \& Adams, R. J. (1993). Marginal maximum likelihood estimation for the ordered partition model. Journal of Educational and Behavioral Statistics, 18(1), 69-90. doi:10.2307/1165183 


\section{Appendix A: Derivations of the ordinal model classes}

Here, we derive and discuss in more detail the ordinal models illustrated in the main tutorial. Throughout, we assume to have observed a total of $N$ values of the ordinal response variable $Y$ with $K+1$ categories from 1 to $K+1$.

\section{Cumulative model}

The cumulative model (CM), sometimes also called graded response model (Samejima, 1997), assumes that the observed ordinal variable $Y$ originates from the categorization of a latent (i.e. not observable) continuous variable $\tilde{Y}$. That is, there are latent thresholds $\tau_{k}$ $(1 \leq k \leq K)$, which partition the values of $\tilde{Y}$ into the $K+1$ observable, ordered categories of $Y$. More formally

$$
Y=k \Leftrightarrow \tau_{k-1}<\tilde{Y} \leq \tau_{k}
$$

for $-\infty=\tau_{0}<\tau_{1}<\ldots<\tau_{K}<\tau_{K+1}=\infty$. We write $\tau=\left(\tau_{1}, \ldots, \tau_{K}\right)$ for the vector of the thresholds. As explained above, it may not be valid to use linear regression on $Y$, because the differences between its categories are not known. However, linear regression is applicable to $\tilde{Y}$. Using $\eta$ to symbolize the predictor term leads to

$$
\tilde{Y}=\eta+\varepsilon,
$$

where $\varepsilon$ is the random error of the regression with $E(\varepsilon)=0$. As we have multiple observations $n$ in our data, it would actually be more explicit to write $Y_{n}, \eta_{n}$, and $\varepsilon_{n}$ in all equations. However, we omit the index $n$ for simplicity and because it is not required to understand the ideas and derivations of the models.

In the simplest case, $\eta$ is a linear predictor of the form $\eta=X b=x_{1} b_{1}+x_{2} b_{2}+\ldots+x_{m} b_{m}$, with $m$ predictor variables $X=\left(x_{1}, \ldots, x_{m}\right)$ and corresponding regression coefficients $b=$ $\left(b_{1}, \ldots, b_{m}\right)$ (without an intercept). The predictor term $\eta$ may also take more complex formsfor instance, multilevel structures or non-linear relationships. However, for the understanding of ordinal models, the exact form of $\eta$ is irrelevant, and we can assume it to be linear for now.

To complete model (15), the distribution $F$ of $\varepsilon$ has to be specified. We might use the normal distribution because it is the default in linear regression, but alternatives such as the logistic distribution are also possible. As explained below, these alternatives are often more appealing then the normal distribution. Depending on the choice of $F$, the final model for $\tilde{Y}$ and also for $Y$ will vary. At this point in the paper, we do not want to narrow down our modeling flexibility and therefore just assume that $\varepsilon_{n}$ is distributed according to $F$ :

$$
\operatorname{Pr}(\varepsilon \leq z)=F(z)
$$

Combining the assumptions (14), (15), and (16) leads to

$$
\begin{aligned}
\operatorname{Pr}(Y \leq k \mid \eta) & =\operatorname{Pr}\left(\tilde{Y} \leq \tau_{k} \mid \eta\right)=\operatorname{Pr}\left(\eta+\varepsilon \leq \tau_{k}\right) \\
& =\operatorname{Pr}\left(\varepsilon \leq \tau_{k}-\eta\right)=F\left(\tau_{k}-\eta\right) .
\end{aligned}
$$


The notation $\mid \eta$ in the first two terms of (17) means the the probabilities will depend on the value of the predictor term $\eta$. Equation (17) says that the probability of $Y$ being in category $k$ or less (depending on $\eta$ ) is equal to the value of the distribution $F$ at the point $\tau_{k+1}-\eta$. In this context, $F$ is also called a response function or processing function. In the present paper, we will use the term distribution and response function interchangeable, when talking about $F$. In case of the CM, $F$ models the probability of the binary outcome $Y \leq k$ against $Y>k$, thus motivating the name "cumulative model".

The probabilities $\operatorname{Pr}(Y=k \mid \eta)$, which are of primary interest, can be easily derived from (17), since

$$
\begin{aligned}
\operatorname{Pr}(Y=k \mid \eta) & =\operatorname{Pr}(Y \leq k \mid \eta)-\operatorname{Pr}(Y \leq k-1 \mid \eta) \\
& =F\left(\tau_{k}-\eta\right)-F\left(\tau_{k-1}-\eta\right) .
\end{aligned}
$$

The CM as formulated in (18) assumes that the predictor term $\eta$ is constant across the response categories. It is plausible that a predictor may have, for instance, a higher impact on the lower categories of an item than on its higher categories. Thus, we could write $\eta_{k}$ to indicate that the predictor term may vary across categories. For instance, if we had 4 response categories and two predictor variables $x_{1}$ and $x_{2}$ with $\eta_{k}=b_{1 k} x_{1}+b_{2 k} x_{2}$, we would have $3 \times 2=6$ regression parameters instead of just 2 . Admittedly, the fully category-specific model is not very parsimonious. Further, estimating regression parameters as varying across response categories in the $\mathrm{CM}$ is not always possible, because it may result in negative probabilities (Tutz, 2000; Van Der Ark, 2001). This can be seen from (18) as follows. If category specific effects are assumed, $\eta_{k}$ may be diferent than $\eta_{k+1}$ and thus

$$
F\left(\tau_{k+1}-\eta_{k+1}\right)-F\left(\tau_{k}-\eta_{k}\right)<0 \quad \text { if } \quad \tau_{k+1}-\eta_{k+1}<\tau_{k}-\eta_{k}
$$

Accordingly, we will have to assume $\eta$ to be constant across categories when using the CM. The threshold parameters $\tau_{k}$, however, are estimated for each category separately, leading to a total of $K$ threshold parameters. This does not mean that it is always necessary to estimate so many of them: We can assume that the distance between two adjacent thresholds $\tau_{k}$ and $\tau_{k+1}$ is the same for all thresholds, which leads to

$$
\tau_{k}=\tau_{1}+(k-1) \delta .
$$

Accordingly, only $\tau_{1}$ and $\delta$ have to be estimated. Parametrizations of the form (20) are often referred to as Rating Scale Models (RSM) (Andersen, 1977; Andrich, 1978b, 1978a) and can be used in many IRT and regression models not only in the CM. When several items each with several categories are administered, this leads to a remarkable reduction in the number of threshold parameters. Consider an example with 7 response categories. Under the model (18) we thus have 6 threshold parameters. Using (20) this reduces to only 2 parameters. The discrepancy will get even larger for an increased number of categories. More details about different parametrizations of the CM can be found, among others, in (Samejima, 1969, $1972,1995,1997)$. Note that in regression models, the threshold parameters are usually of subordinate interest as they only serve as intercept parameters. For this reason, restrictions to $\tau_{k}$ such as (20) are rarely applied in regression models. 


\footnotetext{
${ }^{9}$ The proportional odds assumption can explicitly be tested by comparing the POM when $b$ is constant
ross categories then when it is not (but consider the above described problems of category-specific parameters
the CM). The latter model is often called partial POM (Peterson \& Harrell, 1990). ${ }^{9}$ The proportional odds assumption can explicitly be tested by comparing the POM when $b$ is constant
across categories then when it is not (but consider the above described problems of category-specific parameters
in the CM). The latter model is often called partial POM (Peterson \& Harrell, 1990). ${ }^{9}$ The proportional odds assumption can explicitly be tested by comparing the POM when $b$ is constant
across categories then when it is not (but consider the above described problems of category-specific parameters
in the CM). The latter model is often called partial POM (Peterson \& Harrell, 1990).
} Tutz (2000), which was published in German language only. Originally, the CM was first proposed by Walker and Duncan (1967) but only in the special case where $F$ is the standard logistic distribution, that is where

$$
F(x)=\frac{\exp (x)}{1+\exp (x)}
$$

(see Figure 4, green line). This special model was later called Proportional Odds Model (POM) by McCullagh (1980) and is the most frequently used version of the CM (McCullagh, 1980; Van Der Ark, 2001). In many articles, the CM is directly introduced as the POM and the possibility of using response functions other than the logistic distribution is ignored (Ananth \& Kleinbaum, 1997; Guisan \& Harrell, 2000; Van Der Ark, 2001), thus hindering the general understanding of the CM's ideas and assumptions.

The name of the POM stems from the fact that under this model, the odds ratio of $\operatorname{Pr}\left(Y \leq k_{1} \mid \eta\right)$ against $\operatorname{Pr}\left(Y \leq k_{2} \mid \eta\right)$ for any $1 \leq k_{1}, k_{2} \leq K$ is independent of $\eta$ and only depends on the distance of the thresholds $\tau_{k_{1}}$ and $\tau_{k_{2}}$, which is often called the proportional odds assumption ${ }^{9}$ :

$$
\frac{\operatorname{Pr}\left(Y \leq k_{1} \mid \eta\right) / \operatorname{Pr}\left(Y>k_{1} \mid \eta\right)}{\operatorname{Pr}\left(Y \leq k_{2} \mid \eta\right) / \operatorname{Pr}\left(Y>k_{2} \mid \eta\right)}=\exp \left(\tau_{k_{1}}-\tau_{k_{2}}\right)
$$

Another CM version, the Proportional Hazards Model (PHM), is derived when $F$ is the extreme value distribution (Cox, 1972; McCullagh, 1980):

$$
F(x)=1-\exp (-\exp (x))
$$

(see Figure 4, red line). This model was originally invented in the context of survival analysis for discrete points in time. It is also possible to use the standard normal distribution

$$
F(x)=\Phi(x)=\int_{-\infty}^{x} \frac{1}{\sqrt{2 \pi}} e^{-\frac{z^{2}}{2}} d z .
$$

as a response function (see Figure 4, blue line). Of course, one can use other distributions for $F$ as well.

Following the conventions of generalized linear models, we will often use the name of the inverse distribution function $F^{-1}$, called the link-function, instead of the name of $F$ itself. The link functions associated with the logistic, normal, and extreme value distributions are called logit-, probit, and cloglog-link, respectively. Applying the CM with different response functions to the same data will often lead to similar estimates of the parameters $\tau$ and $b$ as well as to similar model fits (McCullagh, 1980), so that the decision of $F$ usually has only a minor impact on the results.

The derivation and formulation of the general $\mathrm{CM}$ presented in this paper is from 


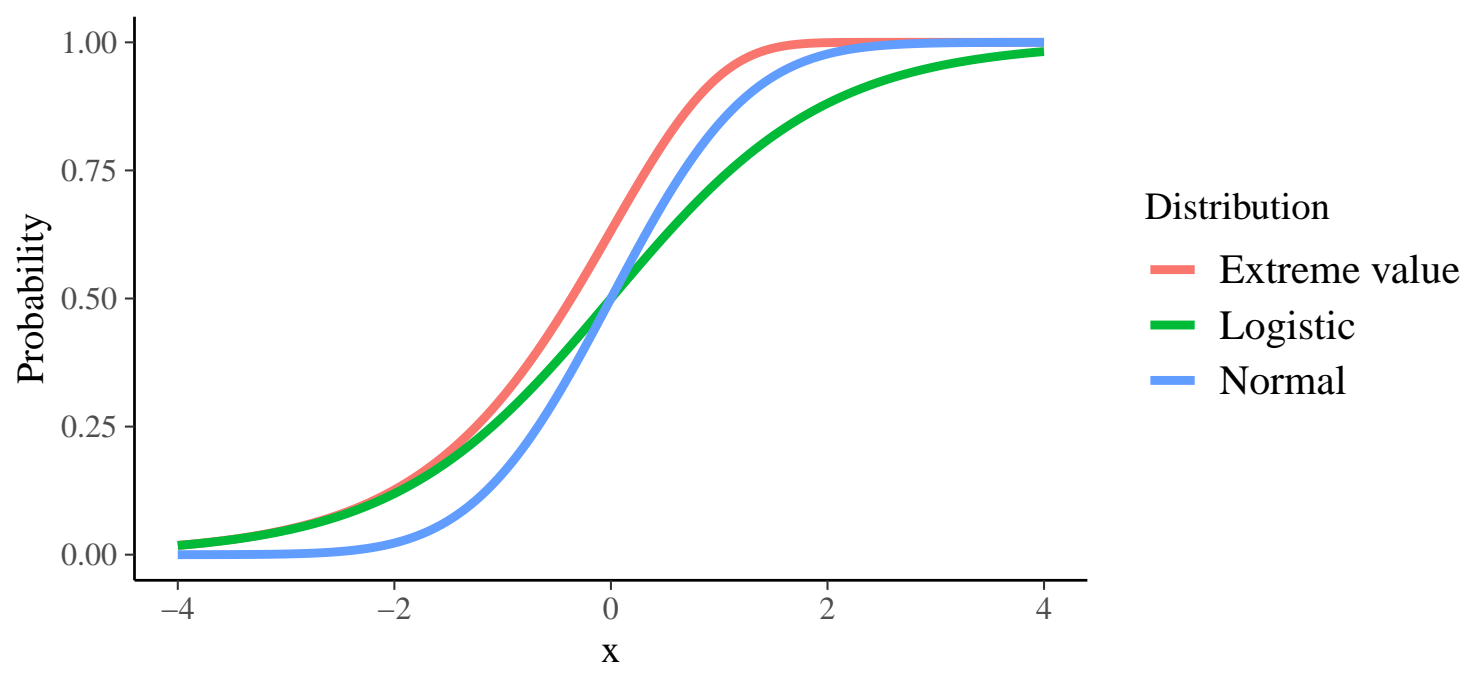

Figure 4. Illustration of various choices for the distribution function $F$.

The derivation of the CM advocated in the present paper demonstrates that this model is especially appealing when the ordinal data $Y$ can be understood as a categorization of a continuous latent variable $\tilde{Y}$, because the thresholds $\tau_{k}$ have an intuitive meaning in this case. However, the CM is also applicable when this assumption seems unreasonable. In particular, the regression parameters $b$ (and inferences about them) remain interpretable in the same way as before (McCullagh, 1980).

\section{Sequential Model}

The dependent variable $Y$ in this model results from a counting process and is truly ordinal in the sense that in order to achieve a category $k$, one has to first achieve all lower categories 1 to $k-1$. The Sequential Model (SM) in its generality proposed by Tutz (1990) explicitly incorporates this structure into its assumptions (see also, Tutz, 2000). For every category $k \in\{1, \ldots, K\}$ there is a latent continuous variable $\tilde{Y}_{k}$ determining the transition between the $k$ th and the $k+1$ th category. The variables $\tilde{Y}_{k}$ may have different meanings depending on the research question. We assume that $\tilde{Y}_{k}$ depends on the predictor term $\eta$ and error $\varepsilon_{k}$ :

$$
\tilde{Y}_{k}=\eta+\varepsilon_{k}
$$

As for the CM, $\varepsilon_{k}$ has mean zero and is distributed according to $F$ :

$$
\operatorname{Pr}\left(\varepsilon_{k} \leq z\right)=F(z)
$$

The sequential process itself is thought as follows: Beginning with category 1 it is checked whether $\tilde{Y}_{1}$ surpasses the first threshold $\tau_{1}$. If not, i.e. if $\tilde{Y}_{1} \leq \tau_{1}$, the process stops and the result is $Y=1$. If $\tilde{Y}_{1}>\tau_{1}$, at least category 2 is achieved (i.e. $Y>1$ ) and the process continuous. Then, it is checked whether $\tilde{Y}_{2}$ surpasses threshold $\tau_{2}$. If not, the process stops with result $Y=2$. Else, the process continues with $Y>2$. Extrapolating this to all 
categories $k \in\{1, \ldots, K\}$, the process stops with result $Y=k$, when at least category $k$ is achieved, but $\tilde{Y}_{k}$ fails to surpass the $k$ th threshold. This event can be written as

$$
Y=k \mid Y \geq k
$$

Combining assumptions (25), (26), and (27) leads to

$$
\begin{aligned}
\operatorname{Pr}(Y=k \mid Y \geq k, \eta) & =\operatorname{Pr}\left(\tilde{Y}_{k} \leq \tau_{k} \mid \eta\right) \\
& =\operatorname{Pr}\left(\eta+\varepsilon_{k} \leq \tau_{k}\right) \\
& =\operatorname{Pr}\left(\varepsilon_{k} \leq \tau_{k}-\eta\right) \\
& =F\left(\tau_{k}-\eta\right) .
\end{aligned}
$$

Equation (28) we can equivalently be expressed by

$$
\operatorname{Pr}(Y=k \mid \eta)=F\left(\tau_{k}-\eta\right) \prod_{j=1}^{k-1}\left(1-F\left(\tau_{j}-\eta\right)\right) .
$$

Because of its derivation, this model is sometimes also called the stopping model. A related sequential model was proposed by Verhelst, Glas, and De Vries (1997) in IRT notation focusing on the logistic response function only. Instead of modeling the probability (28) of the sequential process to stop at category $k$, they suggested to model the probability of the sequential process to continue beyond category $k$. In our notation, this can generally be written as

$$
\operatorname{Pr}(Y \geq k \mid Y \geq k-1, \eta)=F\left(\eta-\tau_{k}\right)
$$

or equivalently

$$
\operatorname{Pr}(Y=k \mid \eta)=\left(1-F\left(\eta-\tau_{k}\right)\right) \prod_{j=1}^{k-1} F\left(\eta-\tau_{j}\right) .
$$

In the following, model (29) is called SMS (short for "sequential model with stopping parameterization") and model (31) is called SMC (short for "sequential model with continuation parameterization"). When $F$ is symmetric, SMS and SMC are identical, because of the relation $F(-x)=1-F(x)$ holding for symmetric distributions. Both, the normal and logistic distribution (24) and (21) are symmetric. Thus, there is only one SM for these distributions. The SM combined with the logistic distribution is often called Continuation Ratio Model (CRM) (Fienberg, 1980, 2007). An example of an asymmetric response function is the extreme value distribution (23). In this case, SMS and SMC are different from each other, but surprisingly, SMS is equivalent to CM (Läärä \& Matthews, 1985). That is, the PHM (Cox, 1972) arises from both, cumulative and sequential modeling assumptions.

Despite their obvious relation, SMS and SMC are discussed independently in two adjacent chapters in the handbook of Linden and Hambleton (1997; see also, Verhelst et al., 1997; Tutz, 1997), leading to the impression of two unrelated models and, possibly, some confusion. This underlines the need of a unified wording and notation of ordinal models, in order to facilitate their understanding and practical use.

In the same way as for the CM, the regression parameters $b$ may depend on the categories when using the SM. In contrast to the CM, however, estimating different regression 
parameters per category is usually less of an issue for the SM (Tutz, 1990, 2000). However, such a model may still be unattractive due to the high number of parameters. Of course, restrictions to the thresholds $\tau_{k}$ such as the rating scale restriction (20) are also applicable. Although the SM is particularly appealing when $Y$ can be understood as the result of a sequential process, it is applicable to all ordinal dependent variables regardless of their origin.

\section{Adjacent Category Model}

The Adjacent Category Model (ACM) is somewhat different than the CM and SM, because, in our opinion, it has no satisfying theoretical derivation. For this reason, we discuss the ideas behind the ACM after introducing its formulas. The ACM is defined as

$$
\operatorname{Pr}(Y=k \mid Y \in\{k, k+1\}, \eta)=F\left(\tau_{k}-\eta\right)
$$

(Agresti, 1984, 2010), that is it describes the probability that category $k$ rather than category $k+1$ is achieved. This can equivalently be written as

$$
\operatorname{Pr}(Y=k \mid \eta)=\frac{\prod_{j=1}^{k-1}\left(1-F\left(\tau_{j}-\eta\right)\right) \prod_{j=k}^{K} F\left(\tau_{j}-\eta\right)}{\sum_{r=1}^{K+1} \prod_{j=1}^{r-1}\left(1-F\left(\tau_{j}-\eta\right)\right) \prod_{j=r}^{K} F\left(\tau_{j}-\eta\right)},
$$

with

$$
\prod_{j=1}^{0}\left(1-F\left(\tau_{j}-\eta\right)\right)=\prod_{j=K+1}^{K} F\left(\tau_{j}-\eta\right):=1
$$

for notational convenience. To our knowledge, the ACM has almost solely been applied with the logistic distribution (21). This combination is the Partial Credit Model (PCM; also called Rasch Rating Model)

$$
\operatorname{Pr}(Y=k \mid \eta)=\frac{\exp \left(\sum_{j=1}^{k-1}\left(\eta-\tau_{j}\right)\right)}{\sum_{r=1}^{K+1} \exp \left(\sum_{j=1}^{r-1}\left(\eta-\tau_{j}\right)\right)}
$$

(with $\sum_{j=1}^{0}\left(\eta-\tau_{j}\right):=0$ ), which is arguably the most widely known ordinal model in psychological research. It was first derived by Rasch (1961) and subsequently by Andersen (1973), Andrich (1978b), Masters (1982), and Fischer (1995) each with a different but equivalent formulation (Adams, Wu, \& Wilson, 2012; Fischer, 1995). Andersen (1973) and Fischer (1995) derived the PCM in an effort to find a model that allows the independent estimation of person and item parameters - a highly desirable property - for ordinal variables. Thus, their motivation for the PCM was purely mathematical and no attempt was made to justify the it theoretically.

On the contrary, Masters (1982) advocated an heuristic approach to the ACM (formulated as the PCM only) by presenting it as the result of a sequential process. In our opinion, his arguments rather lead to the SM than the ACM: The only step that Masters (1982) explains in detail is the last one between category $K$ and $K+1$. For this step, the SMS and the ACM are identical because $(Y \geq K)=(Y \in\{K, K+1\})$. 
Generally modeling the event $Y=k \mid Y \in\{k, k+1\}$ (instead of $Y=k \mid Y \geq k$ ) not only excludes all lower categories 0 to $k-1$, but also all higher categories $k+2$ to $K+1$. When thinking of a sequential process, however, the latter categories should still be achievable after the step to category $k$ was successful. In his argumentation, Masters (1982) explains the last step first and then refers to the other steps as similar to the last step, thus concealing (probably not deliberately) that the PCM is not in full agreement with the sequential process he describes.

Andrich (1978b) and Andrich (2005) presented yet another derivation of the PCM. When two dichotomous processes are independent, four results can occur: $(0,0),(1,0),(0,1),(1,1)$. Using the Rasch model for each of the two processes, the probability of the combined outcome is given by the Polytomous Rasch Model (PRM) (Andersen, 1973; Wilson, 1992; Wilson \& Adams, 1993). When thinking of these processes as steps between ordered categories, $(0,0)$ corresponds to $Y=1,(1,0)$ corresponds to $Y=2$, and $(1,1)$ corresponds to $Y=3$. The event $(0,1)$, however, is impossible because the second step cannot be successful when the first step was not. For an arbitrary number of ordered categories, Andrich (1978b) proved that the PRM becomes the PCM when considering the set of possible events only. While this finding is definitely interesting, it contains no argument that ordinal data observed in scientific experiments may be actually distributed according to the PCM.

Similar to the SM, the threshold parameters $\tau_{k}$ are not necessarily ordered in the ACM, that is the threshold of a higher category may be smaller than the threshold of a lower category. Andrich $(1978 \mathrm{~b}, 2005)$ concluded that this happens when the categories themselves are disordered so that, for instance, category 3 was in fact easier to achieve than category 2. In a detailed logical and mathematical analysis, (Adams et al., 2012) proved the view of Andrich to be incorrect. Instead, this phenomenon is simply a property of the ACM that has no implication on the ordering of the categories.

Despite our criticism, we do not argue that the ACM is worse than the other models. It may not have a satisfying theoretical derivation, but has good mathematical properties especially in the case of PCM. In addition, the same relaxations to the regression and threshold parameters $b$ and $\tau$ can be applied and they remain interpretable in the same way as for the other models, thus making the ACM a valid alternative to the CM and SM.

\section{Generalizations of ordinal models}

An important extention of the ordinal model classes described above is achieved by incorporating a multiplicative effect disc $>0$ (or $\operatorname{disc}_{n}$ to be more explicit) to the terms within the response function $F$. In the cumulative model, for instance, this results in the following model:

$$
\operatorname{Pr}(Y=k \mid \eta, \operatorname{disc})=F\left(\operatorname{disc} \times\left(\tau_{k+1}-\eta\right)\right)-F\left(\operatorname{disc} \times\left(\tau_{k}-\eta\right)\right)
$$

Such an parameter influences the slope of the response function, which may also vary across observations. The higher disc, the steeper the function. It is used in item response theory (IRT) to generalize the 2-Parameter-Logistic (2PL) Model to ordinal data, while the standard 
ordinal models are only generalizations of the 1PL or Rasch model (Rasch, 1961). In this context, we call disc the discrimination parameter. To make sure disc ends up being positive, we often specify its linear predictor $\eta_{\text {disc }}$ on the $\log$-scale so that

$$
\operatorname{disc}=\exp \left(\eta_{\text {disc }}\right)>0 .
$$

We may also use the inverse $s=1$ /disc to model the standard deviation of the latent variables as explained in Section 3.1.2.

\section{Appendix B: Modelling censored years until divorce}

In this section, we continue with the discussion of sequential model to predict years of marriage until divorce. In particular, we will learn how to incorporate censored data into the sequential model. This becomes necessary because - quite fortunately - not all marriages got divorced at the end of the study's observational period.

In the field of time-to-event analysis, the so called hazard rate plays a crucial role (Cox, 1992). For discrete time-to-event data, the hazard rate $h(t)$ at time $t$ is simply the probability that the event occurs at time $t$ given that the event did not occur until time $t-1$. In our notation, the hazard rate at time $t$ can be written as

$$
h(t)=F\left(\tau_{t}-\eta\right)
$$

Comparing this with equation (28; Appendix A), we see that the stopping sequential model is just the product of $h(t)$ and $1-h(t)$ terms for varying values of $t$. Each of these terms defines the event probabilty of a bernoulli variable $(0$ : still married beyond time $t ; 1$ : divorce at time $t$ ) and so the sequential model can be understood as a sequence of conditionally independent bernoulli trials. Accordingly, we can equivalently write the sequential model in terms of binary regression ${ }^{10}$ by expanding each the outcome variable into a sequence of $0 \mathrm{~s}$ and $1 \mathrm{~s}^{11}$. More precisely, for each couple, we create a single row for each year of marriage with the outcome variable being 1 if divorce happened in this year and 0 otherwise. The expanded data is examplified in Table 6 .

In the expanded data set, discrete_time is treated as a factor so that, when included in a model formula, its coefficients will represent the threshold parameters. This can be done in at least two ways. First, we could write ... $0+$ discrete_time $+\ldots$, in which case the coefficients can immediately interpreted as thresholds. Second, we could write ... $\sim 1+$ discrete_time $+\ldots$ so that the intercept is the first threshold, while the $K-1$ coefficients of discrete_time represent differences between the respective other thresholds and the first threshold (dummy coding). Note that these representations are equivalent in

\footnotetext{
${ }^{10}$ Binary regression might be better known as logistic regression, but since we do not apply the logit link in this example, we prefer the former term.

${ }^{11}$ If desired, ordinal sequential models can generally be expressed as generalized liner models (GLMs) and thus fitted with ordinary GLM software. However, this is often much less convenient than directly using the ordinal sequential model, because the data has to be expanded in the way described above. We only recommend using the GLM formulation if the standard formulation is not applicable, for instance when dealing with censored data.
} 
Table 6

Marriage data from the NSFG 2013-2015 survey expanded for use in binary regression.

\begin{tabular}{lllll}
\hline ID & together & age & divorced & discrete_time \\
\hline 1 & yes & 19 & 0.00 & 1 \\
1 & yes & 19 & 0.00 & 2 \\
1 & yes & 19 & 0.00 & 3 \\
1 & yes & 19 & 0.00 & 4 \\
1 & yes & 19 & 0.00 & 5 \\
1 & yes & 19 & 0.00 & 6 \\
1 & yes & 19 & 0.00 & 7 \\
1 & yes & 19 & 0.00 & 8 \\
1 & yes & 19 & 1.00 & 9 \\
2 & yes & 22 & 0.00 & 1 \\
2 & yes & 22 & 0.00 & 2 \\
2 & yes & 22 & 0.00 & 3 \\
2 & yes & 22 & 0.00 & 4 \\
2 & yes & 22 & 0.00 & 5 \\
2 & yes & 22 & 0.00 & 6 \\
2 & yes & 22 & 0.00 & 7 \\
2 & yes & 22 & 0.00 & 8 \\
2 & yes & 22 & 0.00 & 9 \\
\hline
\end{tabular}

the sense that we can transform one into the other. However, the second option usually leads to improved sampling, because it allows brms to do some internal optimization. We are now ready to fit a binary regression model to the expanded data set.

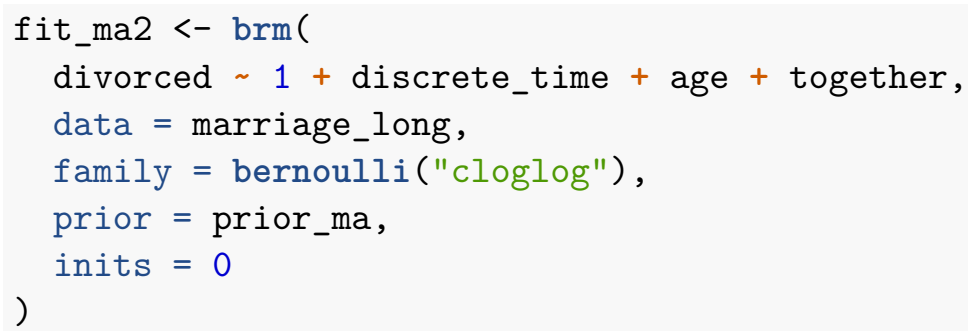

The estimated coefficients of this model are summarized in Table 7. We did not include the threshold estimates in order to keep the table readable. Marginal model predictions are visualized in Figure 5. When interpreting results of the second model, we have to keep in mind that we predicted the probabilty of divorce and not the time of marriage as in the first model. Accordingly, if including the censored data did not change something drastically, we would expect signs of the regression coefficients to be inverted in the second model as compared to the first model. Interestingly, age at marriage (age) has the same sign in both models, leading to opposite conclusions: While the first model predicted longer lasting marriages (lower probability of divorce) for women marrying at lower age, the opposite seems to be true for the second model (probability of divorce was lower for women marrying 


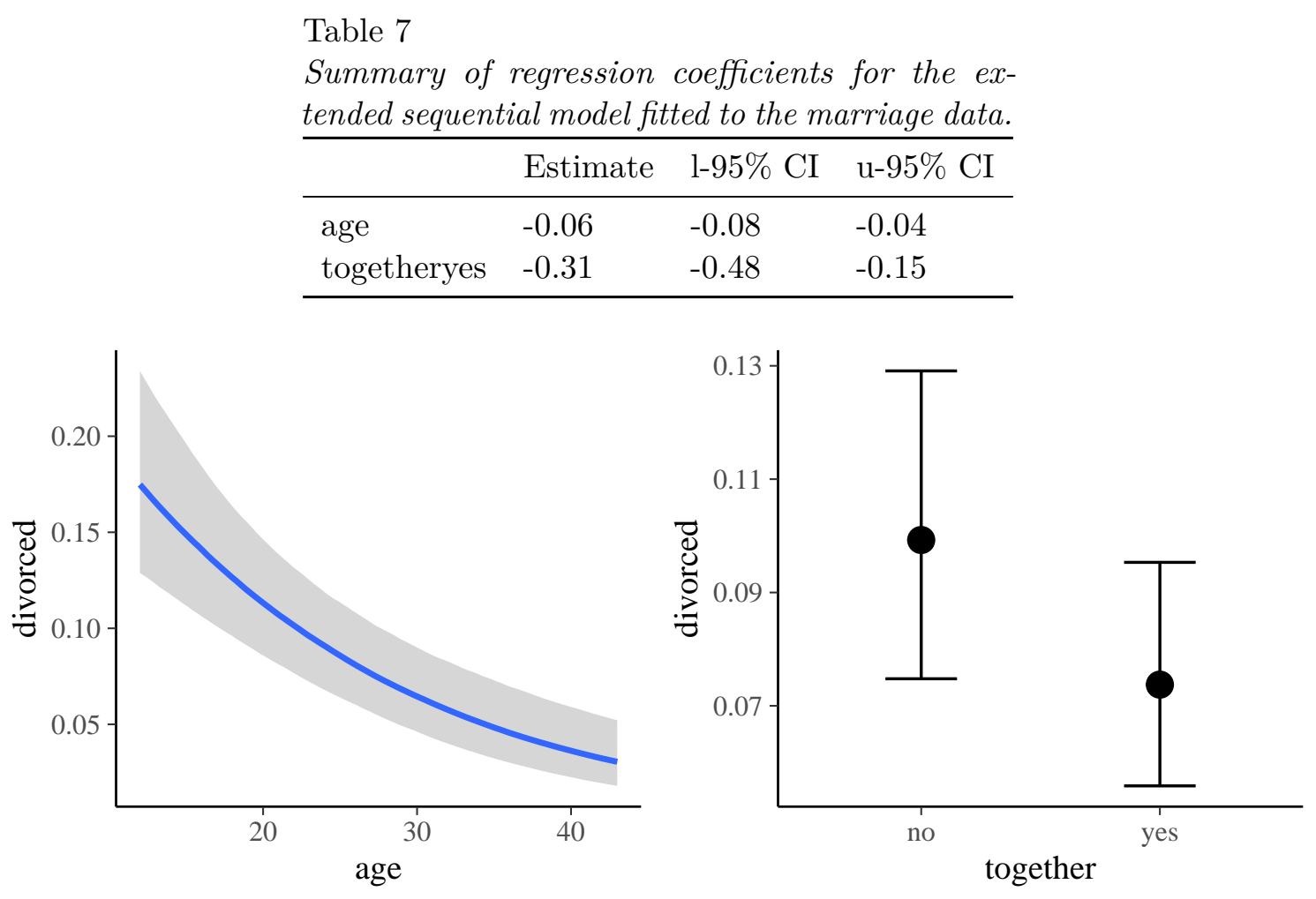

Figure 5. Marginal effects of woman's age at marriage and living together before marriage on the probability of divorce in the 7 th year of marriage.

at older age). This is plausible insofar as censoring is confounded with age at marriage: Women marrying at older ages are more likely to still be married at the time of the survey. Moreover, in contrast to the first model, the second model reveals that couples living together before marriage have considerably lower probability of getting divorced. This underlines the importance of correctly including censored data in (discrete) time-to-event models. The present example has demonstrated how to achieve this in the framework of the ordinal sequential model.

Lastly, we briefly discuss time-varying predictors in discrete time-to-event data. Since the survey took place at one time and asked questions retrospectively, we do not have reliable time-varying predictors for years of marriage, but we can easily think of some potential ones. For instance, the probability of divorce may change over the the duration of marriage with changes in the socio-economic status of the couple. Such time-varying predictors cannot be modeled in the standard sequential model, because all information of a single marriage process has to be stored within the same row in the data set. Fortunately, time-varying predictors can be easily added to the expanded data set shown in Table 6 and then treated in the same way as other predictors in the binary regression model. 